\title{
An innovative procedure to improve integration algorithm for modified Cam-Clay plasticity model
}

\author{
Manouchehr Sanei ${ }^{\mathrm{a}}$, Omar Durán ${ }^{\mathrm{b}}$, Philippe R. B. Devloo ${ }^{\mathrm{c}}$, Erick S.R. \\ Santos $^{\mathrm{d}}$ \\ ${ }^{a}$ Mechanical Engineering Department (FEM) - Petroleum Engineering Division, \\ University of Campinas, Campinas, SP, Brazil \\ ${ }^{b}$ Centre d'Enseignement et de Recherche en Mathématiques et Calcul scientifique, \\ CERMICS (ENPC), Université Paris-Est, Paris, France \\ ${ }^{c}$ Civil Engineering Department (FEC), University of Campinas, Campinas, SP, Brazil \\ ${ }^{d}$ Research and Development Center (Cenpes), Petrobras, Rio de Janeiro, RJ, Brazil
}

\begin{abstract}
An innovative numerical scheme to improve integration algorithm for critical state elastoplasticity is presented and detailed with special consideration to the modified Cam-Clay model. The scheme is based on a rotation of the principal stresses and represent the elastoplastic model in a rotated HaighWestergaard space. Such an approach allows the calculation of modified Cam-Clay to be computationally efficient and easy to implement. The validity of the proposed scheme is verified by comparing the numerical results with analytic solutions.
\end{abstract}

Keywords: Critical state elastoplasticity; Modified Cam-Clay; Integration algorithm; Return mapping; Rotated Haigh-Westergaard space.

\section{Introduction}

Cam-Clay models of critical state soil mechanics are widely applied in various geotechnical applications involving numerical modeling and analysis of soil structure [1]. The original Cam-Clay $(O C C)$ model was presented by Roscoe et al. [2], Schofield \& Wroth [3], and the modified Cam-Clay $(M C C)$

Email address: Corresponding author: manouchehr.sanei@gmail.com (Manouchehr Sanei) 
model was developed by Roscoe \& Burland [4. The general features of CamClay models include pressure sensitivity, hardening and softening responses and demand a small number of material parameters which can be directly obtained from conventional laboratory tests [5]. In particular, the popularity of the $M C C$ model is due to its simplicity and capability to represent the actual behavior of soils [6].

The $M C C$ model depends essentially on two invariants $(P$ and $Q)$. The extension of the $M C C$ model to three-invariant by introducing the Lode's angle $\beta$ was proposed by Alawaji et al. [7. The extended model was represented as the generalized Cam-Clay $(G C C)$ model [8]. The MCC model requires five material parameters, while the $G C C$ model demands six parameters $[8$.

The implementation of the $M C C$ model in finite element demands the use of numerical integration algorithms for presenting the incremental evolution of stresses and hardening parameters [6]. Within the last decade, various integration algorithms have been proposed and categorized within two techniques: explicit and implicit. The implicit algorithms have become predominant because of their efficiency and robustness.

Throughout the years, Borja and his co-authors developed an implicit integration for $M C C$ model by considering either a linear elastic predictor [6] or a nonlinear elastic predictor [9], and later extended the $M C C$ model from the infinitesimal to finite strain [10. In addition, many integration algorithms have been proposed for MCC model, such as documented in [11, 12, 13, 14.

The numerical integration scheme of elastoplastic constitutive models is described in the reference book [15] where the numerical approach is based on the two main steps: the elastic trial step and a subsequent return-mapping scheme. The convergence rate of the iterative method for solving nonlinear elastoplastic equations is strongly dependent on the choice of variables to represent the residual vector. It can be enhanced by representing the elastoplastic equations in terms of the principal stresses [16].

In the present paper, we propose an innovative numerical scheme of the plastic corrector step for $M C C$ model. The scheme is developed by introducing an additional dependence for $M C C$ model on the Lode's angle $\beta$ and representing the $M C C$ model from two-invariant $P-Q$ space to the rotated principal stress (three-invariant) space. Then, we consider a distance function in the rotated Haigh-Westergaard space that should be minimized, in order to compute plastic deformation from closed point projection. The proposed scheme is probably more efficient due to the expression of the yield surface 
within a Haigh-Westergaard cylindrical coordinates [17] and the application of the rotated Haigh-Westergaard space [16].

The proposed approach for the $M C C$ model is verified by comparing the numerical results of $M C C$ model with analytical solutions provided by Perić \& Ayari [8], Perić [18]. The implementation of the present study is done in the NeoPZ library [19], which is an object-oriented scientific computational environment, providing a framework for developing finite element algorithms [20].

\section{Elastoplastic constitutive model}

A nonlinear elastoplastic constitutive model is described by the theory of elastoplasticity, when a material undergoes an irreversible deformations after some specific loading conditions. The total strain tensor $\boldsymbol{\epsilon}$ is decomposed into two parts as follows [15]:

$$
\boldsymbol{\epsilon}=\boldsymbol{\epsilon}_{e}+\boldsymbol{\epsilon}_{p}
$$

where $\boldsymbol{\epsilon}_{e}$ is the elastic component and $\boldsymbol{\epsilon}_{p}$ is the plastic component. The elastic part is reversible and the plastic part represents a permanent deformation, and it is related to the history of irreversible deformations [15. Once a displacement increment $\dot{u}$ is determined, the relationship between strain and displacement under the infinitesimal strain theory is defined as:

$$
\dot{\boldsymbol{\epsilon}}=\frac{1}{2}\left(\nabla \dot{\mathbf{u}}+\nabla^{T} \dot{\mathbf{u}}\right)
$$

The elastoplastic deformation can be as a result of various microstructural changes and it can be described by the set of internal damage variables [21]:

$$
\chi_{i} ; i=1,2, \ldots
$$

where $\boldsymbol{\chi}_{i}$ may be scalars, vectors, or higher rank tensors. With reference to the internal variables, the Helmholtz free energy is defined as in [22]:

$$
\mathbf{F}=\mathbf{F}\left(\boldsymbol{\epsilon}_{e}, \chi_{i}\right)
$$

From the point of view of thermodynamics, the free energy can be decomposed into elastic $\mathbf{F}^{e}\left(\boldsymbol{\epsilon}_{e}\right)$ and plastic $\mathbf{F}^{p}\left(\boldsymbol{\chi}_{i}\right)$ part. By differentiating the elastic part of free energy $\mathbf{F}^{e}$ and substituting the result into the ClausiusDuhem inequality, the expression of stress is written as [21]: 


$$
\boldsymbol{\sigma}=\bar{\rho} \frac{\partial \mathbf{F}^{e}}{\partial \boldsymbol{\epsilon}_{e}}
$$

where $\bar{\rho}$ and $\boldsymbol{\sigma}$ are the mass density and the stress tensor, respectively. Moreover, the thermodynamic conjugate force $\mathbf{A}_{i}$ is represented by:

$$
\mathbf{A}_{i}=\bar{\rho} \frac{\partial \mathbf{F}^{p}}{\partial \boldsymbol{\chi}_{i}}
$$

The elastoplastic deformation is mathematically described by four fundamental axioms as follows [15]:

Elastic law. The elastic law can be presented by two types of constitutive behaviors: linear and nonlinear elasticity. The linear elasticity is described as [23]:

$$
\dot{\boldsymbol{\sigma}}=2 \mu \dot{\boldsymbol{\epsilon}}_{e}+\lambda \operatorname{tr}\left(\dot{\boldsymbol{\epsilon}}_{e}\right) \mathbf{I}
$$

where $\mu$ and $\lambda$ are the Lame coefficients and $\dot{\boldsymbol{\sigma}}$ is the effective stress increment. In many cases the Cam-Clay elastoplastic model is applied to poroelastic materials. In this case the elastoplastic behavior is determined by the value of the effective stress whose relation with the total stress is given by $\boldsymbol{\sigma}_{t}=\boldsymbol{\sigma}-\alpha p \mathbf{I}$ and in which $p$ is the pore pressure and $\alpha$ is the Biot coefficient. The nonlinear stress-strain relationship presented in this paper relates only to the effective stress. No mention to the Biot model will be made in this paper.

Yield criterion. Describes the elastic limit and the plastic part through a plasticity yield function $\Phi=\Phi(\boldsymbol{\sigma}, A)$, where $A=\bar{\rho} \partial \mathbf{F}^{p} / \partial \chi$ is the hardening thermodynamic force and $\chi$ is the internal damage variable. The plasticity function assumes negative values in the elastic part and null values in the plastic part [24].

Flow rule. Assumes the existence of a plastic potential function $\Psi=\Psi(\boldsymbol{\sigma}, A)$, which specifies how the plastic deformation tensor $\boldsymbol{\epsilon}_{p}$ evolves in the plasticity process $\dot{\epsilon}_{p}=\dot{\gamma} \mathbf{N}$, in which $\mathbf{N}(\boldsymbol{\sigma}, A)=\partial \Psi / \partial \boldsymbol{\sigma}$ is the flow direction and $\dot{\gamma}$ is the plastic multiplier. The flow rule is called associative if the plastic potential function equals to yield function, namely $\Psi=\Phi$. For the associative case, the direction of strain rate is the outward normal to yield surface, whereas for non-associative flow rule it is 
the gradient of plastic potential surface [25]. It is required to mention that the $M C C$ model is an associative plasticity model.

Hardening law. Specifies how the internal damage variable $\dot{\chi}=\dot{\gamma} H$ evolves, in which, $H(\boldsymbol{\sigma}, A)=-\partial \Psi / \partial A$ is the hardening modulus.

\subsection{Modified Cam-Clay elastoplasticity model}

The modified Cam-Clay $M C C$ includes four key ingredients: (i) the elastic law, (ii) the yield function, (iii) the flow rule, and (iv) the hardening (softening) law. These four components are defined using the following stress invariants [13]:

$$
\begin{gathered}
P=-\frac{1}{3}\left(\sigma_{1}+\sigma_{2}+\sigma_{3}\right) \\
Q=\left[\frac{1}{2}\left(\sigma_{1}-\sigma_{2}\right)^{2}+\frac{1}{2}\left(\sigma_{2}-\sigma_{3}\right)^{2}+\frac{1}{2}\left(\sigma_{3}-\sigma_{1}\right)^{2}\right]^{\frac{1}{2}}
\end{gathered}
$$

where $P$ is the effective mean stress [MPa], $Q$ is the von Mises equivalent stress [MPa], and $\sigma_{1}, \sigma_{2}$, and $\sigma_{3}$ are the principal of effective stresses [MPa]. Note that $P$ is positive in compression while the general sign conventions are maintained for the Cauchy stress.

\subsubsection{Elastic law of modified Cam-Clay}

In soil mechanics, the $M C C$ model is based on the nonlinear elasticity law that can be linearized (as expressed in the equation (7)) whenever the change of mean effective stress is sufficiently small [15]. The elastic law of $M C C$ establishes a nonlinear relation between the hydrostatic stress (i.e. P) and the volumetric deformation. For the shear stress, two flavours of constitutive relations are commonly used [26]: First, by establishing a constant shear modulus $G[\mathrm{MPa}]$ and Second, by establishing the Poisson's ratio $\nu$ as a constant (in this case $G$ depends on the bulk modulus).

- First. The nonlinear elastic law for the constant shear modulus is:

$$
\boldsymbol{\sigma}-\boldsymbol{\sigma}^{\circ}=2 G\left(\boldsymbol{\epsilon}_{e d}-\boldsymbol{\epsilon}_{e d}^{\circ}\right)-\left(P_{c c}\left(\epsilon_{e v}\right)-P^{o}\right) \mathbf{I}
$$

- Second. The nonlinear elastic law for the constant Poisson's ratio is:

$$
\boldsymbol{\sigma}-\boldsymbol{\sigma}^{\circ}=2 G\left(P_{c c}\left(\epsilon_{e v}\right)\right)\left(\boldsymbol{\epsilon}_{e d}-\boldsymbol{\epsilon}_{e d}^{\circ}\right)-\left(P_{c c}\left(\epsilon_{e v}\right)-P^{o}\right) \mathbf{I}
$$


where the shear modulus for the constant Poisson's ratio is:

$$
G\left(P_{c c}\left(\epsilon_{e v}\right)\right)=\frac{3 K\left(P_{c c}\left(\epsilon_{e v}\right)\right)(1-2 \nu)}{2(1+\nu)}
$$

where $\boldsymbol{\sigma}^{\circ}$ and $\boldsymbol{\sigma}$ are the initial and current effective stress tensor, respectively $[\mathrm{MPa}] . P^{\circ}$ is the initial effective mean stress [MPa]. $\boldsymbol{\epsilon}_{e d}^{\circ}$ and $\boldsymbol{\epsilon}_{e d}$ are the initial and current elastic deviatoric strain, respectively. The elastic volumetric strain $\epsilon_{e v}$ is:

$$
\epsilon_{e v}=\operatorname{tr}\left(\boldsymbol{\epsilon}_{e}\right)
$$

The elastic deviatoric strain tensor $\boldsymbol{\epsilon}_{e d}$ is given by:

$$
\boldsymbol{\epsilon}_{e d}=\boldsymbol{\epsilon}_{e}-\frac{1}{3} \operatorname{tr}\left(\boldsymbol{\epsilon}_{e}\right) \mathbf{I}
$$

The constitutive law for the Cam-Clay effective mean stress $P_{c c}\left(\epsilon_{e v}\right)$ is 27]:

$$
P_{c c}\left(\epsilon_{e v}\right)=-p_{t}+\left(P^{\circ}+p_{t}\right) \exp \left[-\frac{1+e^{\circ}}{C^{e}}\left(\epsilon_{e v}-\epsilon_{e v}^{\circ}\right)\right]
$$

where $e^{\circ}$ is the initial void ratio, $C^{e}$ is the recompression index, $\epsilon_{e v}^{\circ}$ is the initial elastic volumetric strain, and $p_{t}$ is the tensile strength $[\mathrm{MPa}]$. The initial mean effective stress $P^{\circ}$ is represented based on the initial conditions. For a zero tensile strength $P^{\circ}>0$ is required because the $M C C$ constitutive model is generally applied to problems with non-zero initial stresses [26].

The Bulk modulus $K\left(P_{c c}\left(\epsilon_{e v}\right)\right)$ [MPa] in equation 60 is computed as:

$$
K\left(P_{c c}\left(\epsilon_{e v}\right)\right)=-\frac{d P_{c c}\left(\epsilon_{e v}\right)}{d \epsilon_{e v}}
$$

leading to

$$
K\left(\epsilon_{e v}\right)=K\left(P_{c c}\left(\epsilon_{e v}\right)\right)=\frac{1+e^{\circ}}{C^{e}}\left(P_{c c}\left(\epsilon_{e v}\right)+p_{t}\right)
$$


In order to implement the $M C C$ model, it is required to present the elastic tangent operator. The elastic tangent operator $\boldsymbol{C}$ is derived from the derivative of the effective stress tensor in equation (7) with respect to elastic strain tensor. Here, an elastic tangent operator is presented separately for constant shear modulus and constant Poisson's ratio, as:

- The elastic tangent operator for constant shear modulus is:

$$
\boldsymbol{C}=\nabla_{\boldsymbol{\epsilon}_{e}} \boldsymbol{\sigma}=2 G \mathbf{I}^{4}+\left(K\left(\epsilon_{e v}\right)-\frac{2}{3} G\right) \mathbf{I} \otimes \mathbf{I}
$$

- The elastic tangent operator for constant Poisson's ratio is:

$$
\boldsymbol{C}=\nabla_{\boldsymbol{\epsilon}_{e}} \boldsymbol{\sigma}=2 G\left(P_{c c}\left(\epsilon_{e v}\right)\right) \mathbf{I}^{4}+\left(\frac{3 K\left(\epsilon_{e v}\right) \nu}{1+\nu}\right) \mathbf{I} \otimes \mathbf{I}
$$

where $\otimes$ is the tensor product operator, $\mathbf{I}$ is the second-order identity tensor, and $\mathbf{I}^{4}$ is the 4 th-order identity tensor.

- Comparing the elastic tangent operator with the tangent for linear elasticity:

$$
\boldsymbol{C}=\nabla_{\boldsymbol{\epsilon}_{e}} \boldsymbol{\sigma}=2 G \mathbf{I}^{4}+\lambda \mathbf{I} \otimes \mathbf{I}
$$

one can easily identify the equivalent elasticity parameters for a given state of stress or strain.

\subsubsection{Yield function of modified Cam-Clay}

The yield function of the $M C C$ model (Fig. 11) is defined as [15]:

$$
\Phi(P, Q, a)=\left[\frac{P-p_{t}+a}{b a}\right]^{2}+\left[\frac{Q}{M a}\right]^{2}-1
$$

where $M$ is the ratio between the two radii of the $M C C$ ellipse, $a$ is the radius of the ellipse along the effective mean stress axis [MPa]. The parameter $b$ is:

$$
b= \begin{cases}1 & \text { if } P \geq p_{t}-a \\ \zeta & \text { if } P<p_{t}-a\end{cases}
$$

where $\zeta$ is the material constant which modifies the radius of the second half of the ellipse on the compressive side of hydrostatic axis. If $\zeta=1$, the yield 
locus becomes an ellipse with radii $a$ and $M a$, respectively, along $P$ and $Q$. The dashed line in Fig. 1 is named the critical state line. The parameter $M$ is the slope of the critical state line and the area to its right is the supercritical region (Dry, softening) and the area to its left is the subcritical region (Wet, hardening). The critical state line is given by:

$$
Q=M P
$$

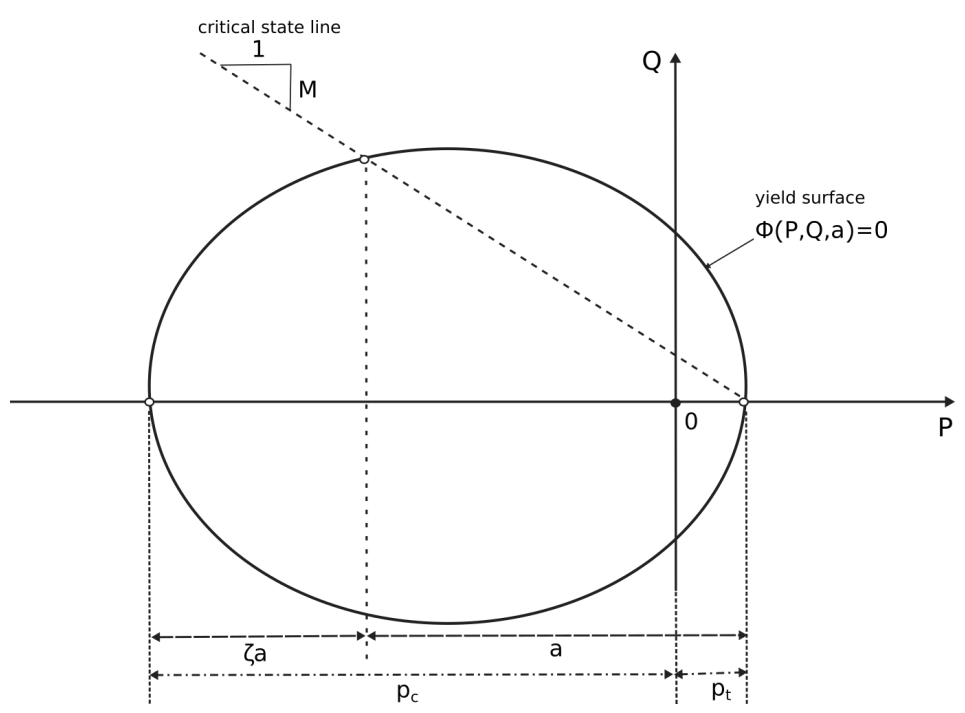

Fig. 1. Modified Cam-Clay $M C C$ model, yield surface [15.

\subsubsection{Flow rule of modified Cam-Clay}

The plastic flow rule is described by postulating associativity, [13] as:

$$
\dot{\epsilon}_{p}=\dot{\gamma} \frac{\partial \Phi}{\partial \boldsymbol{\sigma}}
$$

\subsubsection{Hardening law of modified Cam-Clay}

The hardening law of $M C C$ model is classified as hardening when the yield surface expands $(Q<M P)$, and softening when the surface shrinks $(Q>M P)$ and it undergoes no further changes when $Q=M P$ [10].

The hardening law is expressed by writing the yield surface parameter (a) as a function of hardening variable $(\chi)$. For many plasticity compressible materials, the hardening variable $\chi$ is corresponds to the plastic volumetric strain $\epsilon_{p v}$, namely $\chi \equiv-\epsilon_{p v}$, in which $\epsilon_{p v}=\operatorname{tr}\left(\boldsymbol{\epsilon}_{p}\right)$. The hardening law can 
be defined by a piecewise linear form or an exponential form [26]. The linear form of hardening law is given in terms of preconsolidation pressure $p_{c}[\mathrm{MPa}]$, as follows [15]:

$$
p_{c}(\chi) \equiv(1+\zeta) a(\chi)-p_{t}
$$

The exponential form of the hardening law is written by replacing $a(\chi)$ in equation 25], as follows [27]:

$$
a(\chi) \equiv a\left(-\epsilon_{p v}\right)=a^{\circ} \exp \left[-\frac{\left(1+e^{\circ}\right)}{C^{p}-C^{e}}\left(\epsilon_{p v}-\epsilon_{p v}^{\circ}\right)\right]
$$

where $a^{\circ}$ is the initial value of the hardening parameter, $C^{p}$ is the compression index. $\epsilon_{p v}^{\circ}$ and $\epsilon_{p v}$ are the initial and current plastic volumetric strain, respectively.

\subsection{Yield function of generalized modified Cam-Clay}

The $M C C$ model is generally implemented by a numerical integration of two invariant [18]. Inspired by the articles [7, 28], to control the plastic surface format, we consider a generalized modified Cam-Clay $(G M C C)$ model by introducing an additional dependence on the Lode angle $\beta$. To convert the $M C C$ model in equation (21) from $P-Q$ space to Haigh-Westergaard space, the invariant stress's are expressed as:

$$
P=-\frac{I_{1}}{3} \quad Q=\sqrt{3 J_{2}}
$$

The GMCC model is defined by considering the invariant stress's in equation (27) and applying the Lode's angle $\beta$ as follows:

$$
\Phi\left(I_{1}, \sqrt{J_{2}}, a, \beta\right)=\left[\frac{-\frac{I_{1}}{3}-p_{t}+a}{b a}\right]^{2}+\left[\frac{\sqrt{3} \Gamma(\beta) \sqrt{J_{2}}}{M a}\right]^{2}-1
$$

Here the factor $\Gamma(\beta)$ is given by:

$$
\Gamma(\beta)=\frac{1}{2}\left[(1+\sin (3 \beta))+\frac{1}{\psi}(1-\sin (3 \beta))\right]
$$

where, $\psi$ has the range from $7 / 9$ to $9 / 7$. The $M C C$ model refers to $\psi=1$, such that, $\Gamma(\beta)=1$. 


\subsubsection{Hardening law associated with generalized modified Cam-Clay}

The $M C C$ model is an associative plasticity model where the hardening modulus and flow relation can be related as:

$$
H=-\operatorname{tr}(\boldsymbol{N})
$$

by considering the above property and the definition of incremental plastic deformation $\dot{\epsilon}_{p}=\dot{\gamma} \mathbf{N}$, the relationship for the hardening variable at the plastic correction step can be expressed as:

$$
\dot{\chi}=\dot{\gamma} H=-\dot{\gamma} \operatorname{tr}(\boldsymbol{N})=-\operatorname{tr}\left(\dot{\boldsymbol{\epsilon}}_{p}\right)=-\dot{\epsilon}_{p v}
$$

Then, the incremental plastic volumetric strain while applying the implicit Euler method can be represented by:

$$
\Delta \epsilon_{p v}=\frac{\Delta I_{1}}{3 K\left(\epsilon_{e v}\right)}
$$

where the evaluation of plastic volumetric strain is defined through equations (26), as follow:

$$
\epsilon_{p v}(a)=\epsilon_{p v}^{\circ}+\frac{C^{e}-C^{p}}{1+e^{\circ}} \ln \left(\frac{a}{a^{\circ}}\right)
$$

This derivation will permit the elimination of $\Delta \gamma$ as a variable in returnmapping step.

\section{Numerical integration algorithm for the elastoplastic model}

The numerical integration is divided into two main steps: the elastic trial step and the plastic corrector step (or return-mapping algorithm). If the elastic trial state lies within the elastic domain or on the yield surface, the solution is accepted. Otherwise, if the trial stress in the first step fails to verify the plastic admissible condition, it is projected onto the yield surface by the return-mapping algorithm [15].

\subsection{Incremental elastoplastic constitutive problem (general setting)}

The incremental constitutive model is presented by applying an implicit Euler method. It is formed by giving the elastic deformation $\boldsymbol{\epsilon}_{e}^{n-1}$, the plastic deformation $\boldsymbol{\epsilon}_{p}^{n-1}$, and the hardening variable $\chi^{n-1}$ at a (pseudo) time step $t^{n-1}$, and also given a prescribed incremental strain tensor $\Delta \boldsymbol{\epsilon}$ for the time 
interval $\left[t^{n-1}, t^{n}\right]$ in order to find the following system of algebraic equations at a time-step $t^{n}$ [29, 15]:

$$
\begin{aligned}
& \boldsymbol{\epsilon}_{e}^{n}=\boldsymbol{\epsilon}_{e}^{n-1}+\Delta \boldsymbol{\epsilon}-\Delta \gamma \boldsymbol{N}\left(\boldsymbol{\sigma}^{n}, A^{n}\right) \\
& \chi^{n}=\chi^{n-1}+\Delta \gamma H\left(\boldsymbol{\sigma}^{n}, A^{n}\right)
\end{aligned}
$$

for the unknowns $\boldsymbol{\epsilon}_{e}^{n}, \chi^{n}$ and incremental of plastic multiplier $\Delta \gamma$, it is restricted to the Kuhn-Tucker conditions [30], as:

$$
\Delta \gamma \geq 0, \Phi\left(\boldsymbol{\sigma}^{n}, A^{n}\right) \leq 0, \Delta \gamma \Phi\left(\boldsymbol{\sigma}^{n}, A^{n}\right)=0
$$

where

$$
\boldsymbol{\sigma}^{n}=\left.\bar{\rho} \frac{\partial \mathbf{F}^{e}}{\partial \boldsymbol{\epsilon}_{e}}\right|_{n} \quad A^{n}=A^{n}\left(\chi^{n}\right)=\left.\bar{\rho} \frac{\partial \mathbf{F}^{p}}{\partial \chi}\right|_{n}
$$

and

$$
\mathbf{N}\left(\boldsymbol{\sigma}^{n}, A^{n}\right)=\left.\frac{\partial \Psi}{\partial \boldsymbol{\sigma}}\right|_{n} \quad H\left(\boldsymbol{\sigma}^{n}, A^{n}\right)=-\left.\frac{\partial \Psi}{\partial A}\right|_{n}
$$

Solving the elastoplastic problem occurs in two steps. First a an elastic response is computed (i.e. elastic predictor), where $\Delta \gamma=0$ leading to the elastic trial stress. $\boldsymbol{\epsilon}_{e_{\text {trial }}}^{n}=\boldsymbol{\epsilon}_{e}^{n-1}+\Delta \boldsymbol{\epsilon}$, and internal variables $\chi_{\text {trial }}^{n}=$ $\chi^{n-1} . \quad \boldsymbol{\sigma}_{\text {trial }}^{n}$ and $\Phi\left(\boldsymbol{\sigma}_{\text {trial }}^{n}, A_{\text {trial }}^{n}\right)$ are computed as a function of $\boldsymbol{\epsilon}_{e_{\text {trial }}^{n}}^{n}$. If $\Phi\left(\boldsymbol{\sigma}_{\text {trial }}^{n}, A_{\text {trial }}^{n}\right) \leq 0$, the elastic response is a valid solution and the elasto plastic variables are updated from the trial values $(\cdot)^{n}:=(\cdot)_{\text {trial }}^{n}$. Otherwise, the return-mapping algorithm is applied and a set of nonlinear equations needs to be solved [15]:

$$
\begin{gathered}
\boldsymbol{\epsilon}_{e}^{n}=\boldsymbol{\epsilon}_{e_{\text {trial }}^{n}}^{n}-\Delta \gamma \boldsymbol{N}\left(\boldsymbol{\sigma}^{n}, A^{n}\right) \\
\chi^{n}=\chi_{\text {trial }}^{n}+\Delta \gamma H\left(\boldsymbol{\sigma}^{n}, A^{n}\right) \\
\Delta \gamma>0, \Phi\left(\boldsymbol{\sigma}^{n}, A^{n}\right)=0
\end{gathered}
$$

Once the solution $\boldsymbol{\epsilon}_{e}^{n}$ has been calculated, the plastic strain at a load-step $t^{n}$ can be computed as:

$$
\boldsymbol{\epsilon}_{p}^{n}=\boldsymbol{\epsilon}_{p}^{n-1}+\Delta \boldsymbol{\epsilon}-\Delta \boldsymbol{\epsilon}_{e}
$$

For the particular case of modified Cam-clay model :

$$
\begin{aligned}
\chi^{n} & =-\epsilon_{p v}^{n} \\
\boldsymbol{\sigma}^{n} & =\boldsymbol{\sigma}\left(\boldsymbol{\epsilon}_{e}^{n}\right) \\
\Psi\left(\boldsymbol{\sigma}^{n}\right) & =\Phi\left(\boldsymbol{\sigma}^{n}, \chi^{n}\right) \\
H\left(\boldsymbol{\sigma}^{n}, A^{n}\right) & =\operatorname{tr}\left(\boldsymbol{N}\left(\boldsymbol{\sigma}^{n}, \chi^{n}\right)\right)
\end{aligned}
$$




\subsection{Plastic return-mapping in rotated principal stress}

In order to present a simplified procedure to improve integration scheme for $M C C$ model, a numerical integration algorithm provided by [16] for DiMaggio \& Sandler [31] elastoplastic model is used. The proposed scheme is implemented by using the closest point projection in the rotated principal stresses. In this algorithm, instead of using the six stress components for the representation of the stress state at a point, an alternative representation of the stress is used composed of the principal stresses $\boldsymbol{\sigma}=\left[\sigma_{1}, \sigma_{2}, \sigma_{3}\right]^{\mathrm{T}}$. The principal stresses are then rotated to the Haigh-Westergaard stress space. $\boldsymbol{\sigma}^{*}=\left[\sigma_{1}^{*}, \sigma_{2}^{*}, \sigma_{3}^{*}\right]^{\mathrm{T}}$ is used for the stress representation in the Haigh-Westergaard stress space. The definition of the constitutive model in the rotated principal variables is simpler and computationally more efficient to implement. The coordinate system of rotated principal variables is similar to the decomposition used in [30, 32].

\subsubsection{Haigh-Westergaard stress space}

The stress tensor $\boldsymbol{\sigma}[\mathrm{MPa}]$ sorted in descending order $\sigma_{1}>\sigma_{2}>\sigma_{3}$ can be written as a function of the hydrostatic stress, deviatoric stress, and Lode's angle $(\xi, \rho, \beta)$ [33], as:

$$
\begin{gathered}
\boldsymbol{\sigma}=\left[\begin{array}{l}
\sigma_{1} \\
\sigma_{2} \\
\sigma_{1}
\end{array}\right]=\left[\begin{array}{c}
\frac{1}{\sqrt{3}} \xi+\sqrt{\frac{2}{3}} \rho \cos (\beta) \\
\frac{1}{\sqrt{3}} \xi+\sqrt{\frac{2}{3}} \rho \cos \left(\beta-\frac{2 \pi}{3}\right) \\
\frac{1}{\sqrt{3}} \xi+\sqrt{\frac{2}{3}} \rho \cos \left(\beta+\frac{2 \pi}{3}\right)
\end{array}\right] \\
\xi=\frac{I_{1}}{\sqrt{3}} \quad \rho=\sqrt{2 J_{2}} \quad \beta=\frac{1}{3} \cos ^{-1}\left(\frac{3 \sqrt{2}}{2} \frac{J_{3}}{J_{2}^{3 / 2}}\right)
\end{gathered}
$$

The above expression is valid for the $\beta \in\left[0, \frac{\pi}{3}\right] . I_{1}, J_{2}$, and $J_{3}$ are the first invariant of the stress tensor, the second and third deviatoric stress tensor, respectively. $I_{1}, J_{2}$, and $\beta$ are defined as:

$$
\begin{gathered}
I_{1}=\sigma_{1}+\sigma_{2}+\sigma_{3} \\
J_{2}=\frac{1}{3}\left[\sigma_{1}^{2}+\sigma_{2}^{2}-\sigma_{2} \sigma_{3}+\sigma_{3}^{2}-\sigma_{1}\left(\sigma_{2}+\sigma_{3}\right)\right] \\
\beta=\frac{1}{3} \arccos \left(\frac{\left(2 \sigma_{1}-\sigma_{2}-\sigma_{3}\right)\left(\sigma-2 \sigma_{2}+\sigma_{3}\right)\left(\sigma_{1}+\sigma_{2}-2 \sigma_{3}\right)}{2\left[\sigma_{1}^{2}+\sigma_{2}^{2}-\sigma_{2} \sigma_{3}+\sigma_{3}^{2}-\sigma_{1}\left(\sigma_{2}+\sigma_{3}\right)\right]^{3 / 2}}\right)
\end{gathered}
$$

The relationship between principal stresses and principal strains is expressed as: 


$$
\boldsymbol{\epsilon}=\left[\begin{array}{l}
\epsilon_{1} \\
\epsilon_{2} \\
\epsilon_{3}
\end{array}\right]=\left(\boldsymbol{D}_{H W}\right)^{-1}\left[\begin{array}{l}
\sigma_{1} \\
\sigma_{2} \\
\sigma_{3}
\end{array}\right]
$$

255 where

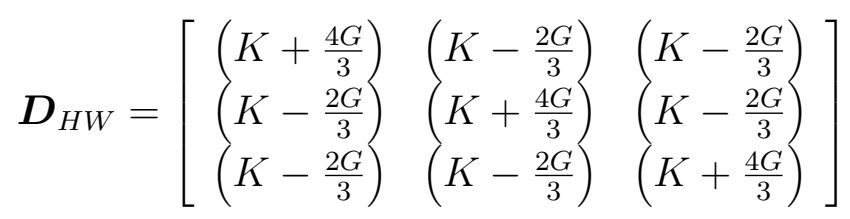

${ }_{256}$ where $K[\mathrm{MPa}]$ and $G[\mathrm{MPa}]$ are the Bulk and the shear modulus, respec257 tively.

263 264

\subsubsection{Rotated Haigh-Westergaard space}

The tensor of stress $\boldsymbol{\sigma}[\mathrm{MPa}]$ can be represented in terms of a rotated Haigh-Westergaard (RHW). The RHW of stress $\boldsymbol{\sigma}^{*}=\left[\sigma_{1}^{*}, \sigma_{2}^{*}, \sigma_{3}^{*}\right]^{T}$ is defined as:

$$
\left[\begin{array}{c}
\sigma_{1}^{*} \\
\sigma_{2}^{*} \\
\sigma_{3}^{*}
\end{array}\right]=\boldsymbol{R}\left[\begin{array}{l}
\sigma_{1} \\
\sigma_{2} \\
\sigma_{3}
\end{array}\right]
$$

where

$$
\boldsymbol{R}=\left[\begin{array}{ccc}
\frac{1}{\sqrt{3}} & \frac{1}{\sqrt{3}} & \frac{1}{\sqrt{3}} \\
\sqrt{\frac{2}{3}} & -\frac{1}{\sqrt{6}} & -\frac{1}{\sqrt{6}} \\
0 & \frac{1}{\sqrt{2}} & -\frac{1}{\sqrt{2}}
\end{array}\right]
$$

The invariant stresses $I_{1}$ and $J_{2}$ and Lode's angle $\beta$ in the rotated space can be computed as:

$$
I_{1}=\sqrt{3} \sigma_{1}^{*} \quad J_{2}=\frac{\sigma_{2}^{* 2}+\sigma_{3}^{* 2}}{2} \quad \beta=\arctan \left(\sigma_{3}^{*} / \sigma_{2}^{*}\right)
$$




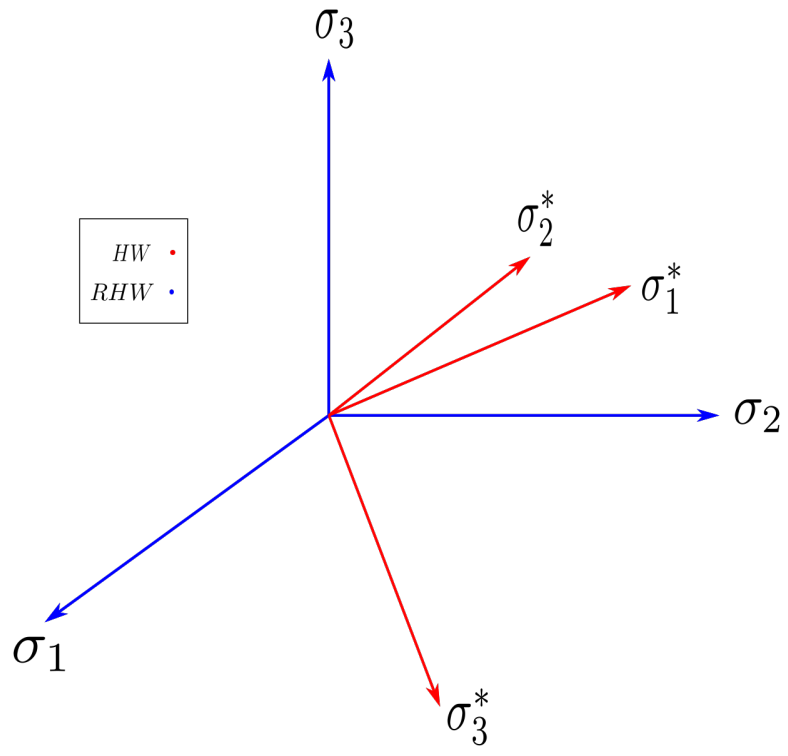

Fig. 2. Transformation of the principal stresses to the rotated Haigh-Westergaard stresses.

The relationship between stress and strain in the rotated Haigh-Westergaard space is:

$$
\left[\begin{array}{l}
\delta \epsilon_{1}^{*} \\
\delta \epsilon_{2}^{*} \\
\delta \epsilon_{3}^{*}
\end{array}\right]=\left(\boldsymbol{D}_{R H W}\right)^{-1}\left[\begin{array}{l}
\delta \sigma_{1}^{*} \\
\delta \sigma_{2}^{*} \\
\delta \sigma_{3}^{*}
\end{array}\right]
$$

where

$$
\boldsymbol{D}_{R H W}=\left[\begin{array}{ccc}
3 K & 0 & 0 \\
0 & 2 G & 0 \\
0 & 0 & 2 G
\end{array}\right]
$$

where $K$ and/or $G$ for nonlinear elasticity are a function of $\epsilon_{e v}$.

\subsection{Incremental elastoplastic constitutive problem in $R H W$ space}

The incremental associative elastoplastic problem can be interpreted using a distance minimization of the trial stress to the admissibility surface in the RHW space [34, 35]. The admissibility surface for a given variable 
$\chi$ is $S^{*}(\chi)=\left\{\boldsymbol{\sigma}^{*}: \Phi^{*}\left(\boldsymbol{\sigma}^{*}, A(\chi)=0\right)\right\}$, where $\Phi^{*}\left(\boldsymbol{\sigma}^{*}, A\right)$ is the yield function. The expression of the return map in the RHM space corresponding to equation $(38)$ is:

$$
\begin{gathered}
\boldsymbol{\epsilon}_{e_{\text {trial }}^{* n}}^{* n}-\boldsymbol{\epsilon}_{e}^{*, n}=\Delta \gamma \boldsymbol{N}^{*, n} \\
\chi_{\text {trial }}^{n}-\chi^{n}=-\Delta \gamma H^{*, n}
\end{gathered}
$$

where the flow direction $N^{*}$ is:

$$
\boldsymbol{N}^{*, n}=\frac{\partial \Phi^{*}\left(\boldsymbol{\sigma}^{*, n}, A\left(\chi^{n}\right)\right)}{\partial \boldsymbol{\sigma}^{*}}
$$

and the hardening modulus $H^{*}$ is:

$$
H^{*, n}=-\frac{\partial \Phi^{*}\left(\boldsymbol{\sigma}^{*, n}, A\left(\chi^{n}\right)\right)}{\partial A}
$$

Considering that the direction $\boldsymbol{N}^{*, n}$ is orthogonal to the surface $S^{*}(\chi)$, the inner product of a variation of $\delta \boldsymbol{\sigma}^{*}$ along the tangent plane at $\boldsymbol{\sigma}^{*, n} \in$ $S^{*}\left(\chi^{n}\right)$ should satisfy the following orthogonality relationship:

$$
\begin{gathered}
0=\left(\delta \boldsymbol{\sigma}^{*}, \boldsymbol{\epsilon}_{\text {trial }}^{*, n}-\boldsymbol{\epsilon}^{*, n}\right)=\left(\delta \boldsymbol{\sigma}^{*}, \boldsymbol{D}_{R H W}^{-1}\left(\boldsymbol{\sigma}_{\text {trial }}^{*, n}-\boldsymbol{\sigma}^{*, n}\right)\right)= \\
\left\langle\delta \boldsymbol{\sigma}^{*}, \boldsymbol{\sigma}_{\text {trial }}^{*, n}-\boldsymbol{\sigma}^{*, n}\right\rangle_{R H W}
\end{gathered}
$$

where $\boldsymbol{\sigma}^{*, n}$ is the projected point in $S^{*}\left(\chi^{n}\right)$. Define the internal product for vectors $\mathbf{a}^{*}$ and $\mathbf{b}^{*}$ in the RHW space as:

$$
\left\langle\mathbf{a}^{*}, \mathbf{b}^{*}\right\rangle_{R H W}=\left(\mathbf{a}^{*}, \boldsymbol{D}_{R H W}^{-1} \mathbf{b}^{*}\right)
$$

The equation (54) is a distance function which shows minimizing the distance induced by the RHW internal product of the trial stress to the admissibility surface. The distance function for nonlinear elasticity can be written as:

$$
d\left(\mathbf{a}^{*}, \mathbf{b}^{*}\right):=\left\{\frac{\left(\mathbf{a}_{1}^{*}-\mathbf{b}_{1}^{*}\right)^{2}}{3 K\left(\epsilon_{e v}\right)}+\frac{\left(\mathbf{a}_{2}^{*}-\mathbf{b}_{2}^{*}\right)^{2}}{2 G\left(\epsilon_{e v}\right)}+\frac{\left(\mathbf{a}_{3}^{*}-\mathbf{b}_{3}^{*}\right)^{2}}{K\left(\epsilon_{e v}\right)}\right\}^{1 / 2}
$$

where the function $K\left(\epsilon_{e v}\right)$ depends on the elastic volumetric strain and the function $G\left(\epsilon_{e v}\right)$ depends on the flavour of the elastic constitutive law (i.e. $G$ constant or $\nu$ constant). It means: 


$$
G\left(\epsilon_{e v}\right)=\left\{\begin{array}{l}
\text { G constant : } \\
\nu \text { constant }: \quad G\left(\epsilon_{e v}\right)=\frac{3 K\left(P_{c c}\left(\epsilon_{e v}\right)\right)(1-2 \nu)}{2(1+\nu)}
\end{array}\right.
$$

The projected point, which minimizes the aforementioned distance function is computed as:

$$
\boldsymbol{\sigma}^{*, n}=\min _{\boldsymbol{\sigma}^{*, n} \in S^{*}\left(\chi^{n}\right)} d\left(\boldsymbol{\sigma}_{\text {trial }}^{*, n}, \boldsymbol{\sigma}^{*, n}\right)
$$

\subsection{Solution of the incremental return-mapping step}

In the RHW space, a stress point on the yield surface depends on three parameters as:

$$
S^{*}\left(\chi^{n}\right)=\left\{\boldsymbol{\sigma}^{*, n}\left(r_{1}, r_{2}, \chi^{n}\right):\left(r_{1}, r_{2}, \chi^{n}\right) \in \mathcal{I}\right\}
$$

where the internal damage parameter $\chi^{n}$ is included to define the position of the surface and the other two parameters $r_{1}$ and $r_{2}$ which determine the position of a point on the surface. The square distance of a given $\boldsymbol{\sigma}_{\text {trial }}^{*, n}$, to a point on the surface $\boldsymbol{\sigma}^{*, n}\left(r_{1}, r_{2}, \chi^{n}\right) \in S^{*}\left(\chi^{n}\right)$ is defined as:

$$
\delta\left(\boldsymbol{\sigma}_{\text {trial }}^{*, n} ; r_{1}, r_{2}, \chi^{n}\right)=\left(d\left(\boldsymbol{\sigma}_{\text {trial }}^{*, n}, \boldsymbol{\sigma}^{*, n}\left(r_{1}, r_{2}, \chi^{n}\right)\right)\right)^{2}
$$

where $d(\cdot)$ is defined in equation (56).

Given the trial elastic strain $\boldsymbol{\epsilon}_{\text {trial }}^{*, n}$ and internal variable $\chi_{\text {trial }}^{n}=\chi^{n-1}$, the quantity of $\boldsymbol{\sigma}_{\text {trial }}^{*, n}$ is computed as:

$$
\boldsymbol{\sigma}_{\text {trial }}^{n}=\boldsymbol{\sigma}_{\text {trial }}^{n-1}+2 G\left(\epsilon_{\text {ev }}^{n}\right) \boldsymbol{\epsilon}_{e d_{\text {trial }}}^{n}-2 G\left(\epsilon_{\text {ev }}^{n-1}\right) \boldsymbol{\epsilon}_{e d_{\text {trial }}}^{n-1}-\left(P_{c c}\left(\epsilon_{\text {ev }}^{n}\right)-P_{c c}\left(\epsilon_{e v}^{n-1}\right)\right) \mathbf{I}
$$

The relation between a stress increment and a strain increment for the Cam-clay nonlinear elasticity in the rotated Haigh-Westergaard space as documented in equation (50) is:

$$
\boldsymbol{D}_{R H W}\left(\epsilon_{e v}\right)=\left[\begin{array}{ccc}
3 K\left(\epsilon_{e v}\right) & 0 & 0 \\
0 & 2 G\left(\epsilon_{e v}\right) & 0 \\
0 & 0 & 2 G\left(\epsilon_{e v}\right)
\end{array}\right]
$$

where the expression for $G\left(\epsilon_{e v}\right)$ depends on the flavour of the nonlinear elastic law. It is required to mention that the Bulk modulus and shear modulus in equation (61) are the functions of elastic volumetric strain that are belong 
to trial stress tensor. Then, in the rest of paper, the following notations are applied.

$$
\left\{\begin{array}{l}
K_{\text {trial }}=K\left(\epsilon_{e v_{\text {trial }}}\right) \\
G_{\text {trial }}=G\left(\epsilon_{\text {ev }}\right)
\end{array}\right.
$$

After computing the quantity of $\boldsymbol{\sigma}_{\text {trial }}^{*, n}$, the stress $\boldsymbol{\sigma}^{*, n}\left(r_{1}, r_{2}, \chi^{n}\right) \in S^{*}\left(\chi^{n}\right)$ at a time-step $t^{n}$ is computed by minimizing the distance of $\boldsymbol{\sigma}_{\text {trial }}^{*, n}$ to the admissibility surface $S^{*}\left(\chi^{n}\right)$, as follows:

$$
\begin{aligned}
& \frac{\partial \delta\left(\sigma_{\text {trial }}^{*, n} ; r_{1}, r_{2}, \chi^{n}\right)}{\partial r_{1}} \quad=0 \\
& \frac{\partial \delta\left(\sigma_{\text {trial }}^{*, n} ; r_{1}, r_{2}, \chi^{n}\right)}{\partial r_{2}} \quad=0 \\
& \chi^{n}-\chi^{n-1}-\Delta \gamma H^{*}\left(r_{1}, r_{2}, \chi^{n}\right)=0 \\
& \boldsymbol{\epsilon}_{e_{\text {trial }}^{*, n}}-\boldsymbol{\epsilon}_{e}^{*, n}-\Delta \gamma \boldsymbol{N}^{*}\left(r_{1}, r_{2}, \chi^{n}\right)=0
\end{aligned}
$$

For the particular case of modified Cam-clay model, the system of equations (63) when considering the equation (31), can be simplified as:

$$
\begin{array}{cc}
\frac{\partial \delta\left(\sigma_{\text {trial }}^{*, n} ; r_{1}, r_{2}, \chi^{n}\right)}{\partial r_{1}} & =0 \\
\frac{\partial \delta\left(\sigma_{\text {trial }}^{*,} ; r_{1}, r_{2}, \chi^{n}\right)}{\partial r_{2}} & =0 \\
\chi^{n}-\chi^{n-1}+\epsilon_{p v}^{n}-\epsilon_{p v}^{n-1} & =0
\end{array}
$$

which is a system of equation of three equations in three unknowns.

\subsection{The generalized modified Cam-Clay in the RHW space}

The GMCC surface in the RHW space as shown in Fig. 3 is formed by two parts. There is one softening part $S_{s}^{*}$ (Green color) and one hardening part $S_{h}^{*}$ (Red color). The implementation of the return map to any of these surfaces is similar and the only difference is related to the quantity of $b$, where $b=1.0$ for softening part and $b=\zeta$ for the hardening part. Therefore, only the return mapping to the hardening part of $G M C C$ in the RHW space is discussed. 


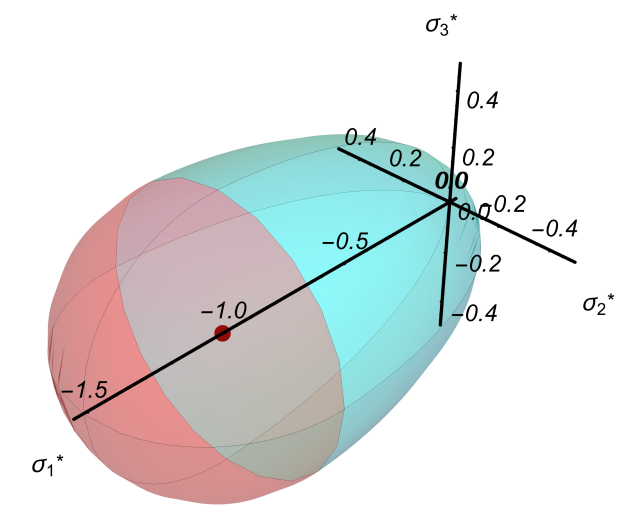

Fig. 3. Yield surface represented in the RHW stress space: softening part $S_{s}^{*}$ with green color and hardening part $S_{h}^{*}$ with red color.

The RHW representation of any point on the hardening surface $S_{h}^{*}$ of the GMCC model can be represented in term of three parameters $r_{1}=\theta, r_{2}=\beta$, and $r_{3}=a$. That is:

$$
\left[\begin{array}{l}
\sigma_{1}^{*} \\
\sigma_{2}^{*} \\
\sigma_{3}^{*}
\end{array}\right]=\left[\begin{array}{c}
\frac{3(a+a b \cos (\theta))}{\sqrt{3}} \\
\cos (\beta) \frac{\sqrt{2} F(a) \sin (\theta)}{\Gamma(\beta)} \\
\sin (\beta) \frac{\sqrt{2} F(a) \sin (\theta)}{\Gamma(\beta)}
\end{array}\right]
$$

where $F(a)$ is defined by the following expression:

$$
F(a)=\frac{M \sqrt{-p_{t}\left(2 a b+p_{t}\right)}}{\sqrt{3} b \Gamma(\beta)}
$$

\subsection{Numerical integration algorithm for generalized modified Cam-Clay}

The minimization of RHW square distance from $\boldsymbol{\sigma}_{\text {trial }}^{*, n}$ to a point $\boldsymbol{\sigma}^{*, n} \in$ $S_{h}^{*}(a)$ for $G M C C$ model is presented for linear and nonlinear elasticity. In the rest of paper, for the simplicity of notation, we will use the notation $\boldsymbol{\sigma}_{\text {trial }}^{*}=\boldsymbol{\sigma}_{\text {trial }}^{*, n}$ and $\boldsymbol{\sigma}^{*, n}=\boldsymbol{\sigma}^{*}$. Using the definition (65), the RHW square distance for nonlinear elasticity is: 


$$
\begin{gathered}
d^{2}\left(\boldsymbol{\sigma}_{\text {trial }}^{*}, \boldsymbol{\sigma}^{*}\right)=\frac{\left(\sigma_{\text {trial }, 1}^{*}-\frac{3(a+a b \cos (\theta))}{\sqrt{3}}\right)^{2}}{3 K_{\text {trial }}}+\frac{\left(\sigma_{\text {trial }, 2}^{*}-\cos (\beta) \frac{\sqrt{2} F(a) \sin (\theta)}{\Gamma(\beta)}\right)^{2}}{2 G_{\text {trial }}}+ \\
\frac{\left(\sigma_{\text {trial }, 3}^{*}-\sin (\beta) \frac{\sqrt{2} F(a) \sin (\theta)}{\Gamma(\beta)}\right)^{2}}{2 G_{\text {trial }}}:=\delta_{h}\left(\boldsymbol{\sigma}_{\text {trial }}^{*} ; \theta, \beta, a\right)
\end{gathered}
$$

where the expression for $G_{\text {trial }}$ depends on the flavor of the nonlinear elastic law.

The third relation of equation $(64)$ by considering the equation 32 can be written as:

$$
I_{1}\left(\boldsymbol{\sigma}_{\text {trial }}^{*}\right)-I_{1}\left(\boldsymbol{\sigma}^{*}\right)=3 K_{\text {trial }}\left[\epsilon_{p v}\left(a_{\text {trial }}\right)-\epsilon_{p v}(a)\right]
$$

The equation $(68)$ is applied to update simultaneously the position of the yield surface which is defined by the variable $a$. In the RHW space, the invariant $I_{1}\left(\boldsymbol{\sigma}^{*}\right)$ is expressed by:

$$
I_{1}\left(\boldsymbol{\sigma}^{*}\right)=I_{1}(\theta, a)=3(a+a b \cos (\theta))
$$

Define a residual associated with the position of the cap:

$$
\operatorname{res}(\theta, a)=\left[I_{1}\left(\boldsymbol{\sigma}_{\text {trial }}^{*}\right)-I_{1}(\theta, a)\right]-3 K_{\text {trial }}\left[\varepsilon_{p v}\left(a_{\text {trial }}\right)-\varepsilon_{p v}(a)\right]=0
$$

The projected point on the Cam-clay surface as a function of $a_{\text {trial }}$ and $\boldsymbol{\sigma}_{\text {trial }}^{*}$ can be obtained by computing $a$ and $\boldsymbol{\sigma}_{\text {proj }}^{*} \in S_{h}^{*}(a)$ which minimizes the RHW distance to $\boldsymbol{\sigma}_{\text {trial }}^{*}$. The point is found by applying Newton's method to find the values of $\theta, \beta$, and $a$ such that:

$$
\boldsymbol{R}_{h}=\left[\begin{array}{c}
\frac{\partial \delta_{h}\left(\boldsymbol{\sigma}_{\text {trial }}^{*} ; \theta, \beta, a\right)}{\partial \theta} \\
\frac{\partial \delta_{h}\left(\boldsymbol{\sigma}_{\text {trial }}^{*} ; \theta, \beta, a\right)}{\partial \beta} \\
\operatorname{res}(\theta, a)
\end{array}\right]=\left[\begin{array}{l}
0 \\
0 \\
0
\end{array}\right]
$$

The procedure to solve the system of equations, derivative of the projected stresses, and the derivation of elastoplastic tangent operator for this $G M C C$ model is documented in the Appendices A, B, and C. 


\section{Numerical results}

To verify the implementation of $G M C C$ model, we compare the numerical results of our implementation with the analytical solution provided by Perić [18] for infinitesimal strain assumption. The analytical solutions are presented separately for volumetric and deviatoric behavior of the material.

\subsection{The volumetric behavior}

The rate of volumetric strain is obtained from its nonlinear elasticity and hardening rule. Then, by integrating the rate over a finite time increment, the analytical expressions for volumetric elastic strain and plastic strain are derived as:

$$
\begin{gathered}
\epsilon_{e v}=\epsilon_{e v}^{\circ}+\frac{1}{1+e^{\circ}} \ln \left[\left(\frac{P}{P^{\circ}}\right)^{-C^{e}}\right] \\
\epsilon_{p v}=\epsilon_{p v}^{\circ}+\frac{1}{1+e^{\circ}} \ln \left[\left(\left(\frac{P}{P^{\circ}}\right)\left(\frac{M^{2}+\bar{\eta}^{2}}{M^{2}+\bar{\eta}_{\circ}^{2}}\right)\right)^{C^{e}-C^{p}}\right]
\end{gathered}
$$

where $\bar{\eta}$ is the modified stress ratio and defines as:

$$
\bar{\eta}=\eta g \quad \eta=\frac{Q}{P}
$$

In the case of a conventional triaxial compression, the function $g$ is equal to 1.0. The superscript $\left(^{\circ}\right)$ refers to the initial condition herein.

A total volumetric strain is:

$$
\epsilon_{v}=\epsilon_{v}^{\circ}+\frac{1}{1+e^{\circ}} \ln \left[\left(\frac{P}{P^{\circ}}\right)^{-C^{p}}\left(\frac{M^{2}+\bar{\eta}^{2}}{M^{2}+\bar{\eta}_{\circ}^{2}}\right)^{C^{e}-C^{p}}\right]
$$

By considering a stress path in $P-Q$ space, with a slope $\bar{k}=k g$, in which $k=Q / P$, the total volumetric strain is expressed as:

$$
\epsilon_{v}=\epsilon_{v}^{\circ}+\frac{1}{1+e^{\circ}} \ln \left[\left(\frac{\bar{k}-\bar{\eta}_{\circ}}{\bar{k}-\bar{\eta}}\right)^{-C^{p}}\left(\frac{M^{2}+\bar{\eta}^{2}}{M^{2}+\bar{\eta}_{\circ}^{2}}\right)^{C^{e}-C^{p}}\right]
$$

Note that in the case of conventional triaxial test $\bar{k}=3.0$. 


\subsection{The deviatoric behavior}

By integrating the rate of deviatoric strain over a finite time increment, the analytical expressions for generalized shear elastic strain and plastic strain are obtained as:

$$
\begin{gathered}
\epsilon_{e q}=\epsilon_{e q}^{\circ}+\frac{1}{1+e^{\circ}} \ln \left[\left(\frac{\bar{k}-\bar{\eta}}{\bar{k}-\bar{\eta}_{\circ}}\right)^{-\frac{C^{e} \bar{k}}{3 \omega g}}\right] \\
\epsilon_{p q}=\epsilon_{p q}^{\circ}-\frac{2 C_{i}}{M\left(1+e^{\circ}\right)}\left[\arctan \left(\frac{\bar{\eta}}{M}\right)-\arctan \left(\frac{\bar{\eta}_{\circ}}{M}\right)\right]+ \\
\frac{1}{1+e^{\circ}} \ln \left[\left(\frac{M-\bar{\eta}}{M-\bar{\eta}_{\circ}}\right)^{\frac{C_{i} \bar{k}}{M(M-\bar{k})}}\left(\frac{M+\bar{\eta}}{M+\bar{\eta}_{\circ}}\right)^{\frac{C_{i} \bar{k}}{M(M+\bar{k})}}\left(\frac{\bar{k}-\bar{\eta}}{\bar{k}-\bar{\eta}_{\circ}}\right)^{\frac{2 C_{i} \bar{k}}{\bar{k}^{2}-M^{2}}}\right]
\end{gathered}
$$

where

$$
C_{i}=\left(C^{p}-C^{e}\right) g \quad \omega=\frac{3(1-2 \nu)}{2(1+\nu)}
$$

A total generalized shear strain is:

$$
\begin{gathered}
\epsilon_{q}=\epsilon_{q}^{\circ}-\frac{2 C_{i}}{M\left(1+e^{\circ}\right)}\left[\arctan \left(\frac{\bar{\eta}}{M}\right)-\arctan \left(\frac{\bar{\eta}_{\circ}}{M}\right)\right]+ \\
\frac{1}{1+e^{\circ}} \ln \left[\left(\frac{M-\bar{\eta}}{M-\bar{\eta}_{\circ}}\right)^{\frac{C_{i} \bar{k}}{M(M-\bar{k})}}\left(\frac{M+\bar{\eta}}{M+\bar{\eta}_{\circ}}\right)^{\frac{C_{i} \bar{k}}{M(M+\bar{k})}}\left(\frac{\bar{k}-\bar{\eta}}{\bar{k}-\bar{\eta}_{\circ}}\right)^{\frac{2 C_{i} \bar{k}}{\bar{k}^{2}-M^{2}}-\frac{C^{e} \bar{k}}{3 \omega g}}\right]
\end{gathered}
$$

The axial and radial strains in a triaxial test can be calculated from volumetric and generalized shear strains as follows:

$$
\epsilon_{a}=\frac{1}{3} \epsilon_{v}+\epsilon_{q} \quad \epsilon_{r}=\frac{1}{3} \epsilon_{v}-\frac{1}{2} \epsilon_{q}
$$

\subsection{Verification of the implementation}

The consolidated-drained triaxial test documented in [36] is reproduced. In this test, the sample is first consolidated under hydrostatic pressure and thus sheared by applying additional axial load. The material parameters that were used in this test are listed in Table 1. The test is on a normally consolidated clay sample which involves elastoplastic loading, a behavior that considers hardening. The stress paths, initial and final yield surfaces for softening and hardening part of this test is shown in Fig. 4. The performance of this test is done in two examples. First by giving the constant shear modulus and second by considering the constant Poisson's ratio. 
Table 1. Material parameters for clay [36].

\begin{tabular}{|c|c|}
\hline Parameter & Value \\
\hline \hline$G$ (Shear modulus) & $20 \mathrm{MPa}$ \\
\hline$\nu$ (Poisson's ratio) & 0.3 \\
\hline$M$ & 1.2 \\
\hline$C^{e}$ & 0.0077 \\
\hline$C^{p}$ & 0.066 \\
\hline$e^{\circ}$ & 0.438 \\
\hline$\psi$ & 1 \\
\hline$P^{\circ}$ & $0.2 \mathrm{MPa}$ \\
\hline$p_{c}^{\circ}$ & $0.2 \mathrm{MPa}$ \\
\hline$Q^{\circ}$ & $0.0 \mathrm{MPa}$ \\
\hline
\end{tabular}

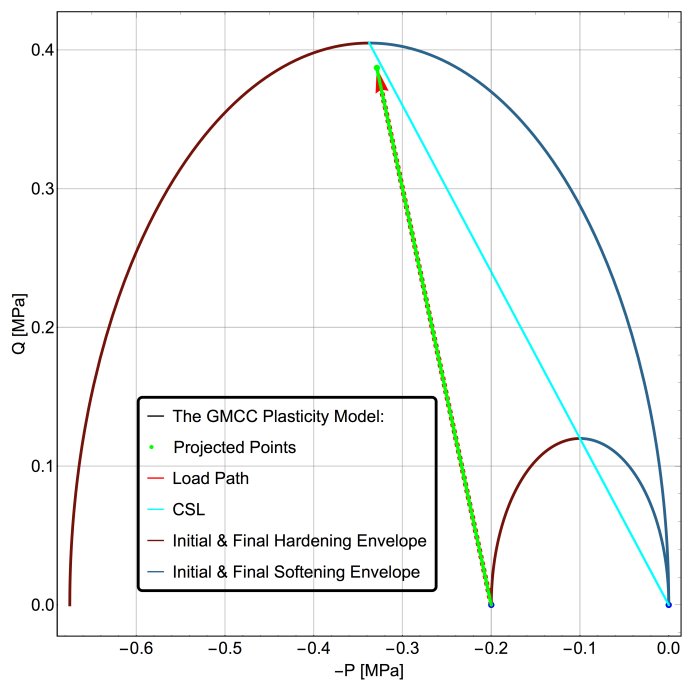

Fig. 4. The normally consolidated-drained triaxial test: stress paths, initial and final yield surfaces for softening and hardening part in the $P-Q$ space.

\subsubsection{Verification for constant shear modulus}

For the following triaxial test, all the subfigures in the Fig. 5 presents a comparison for the $G M C C$ model implementation with the results calculated from an analytical solution (see in the Appendix D), displaying the verification of the implementation for constant shear modulus. 

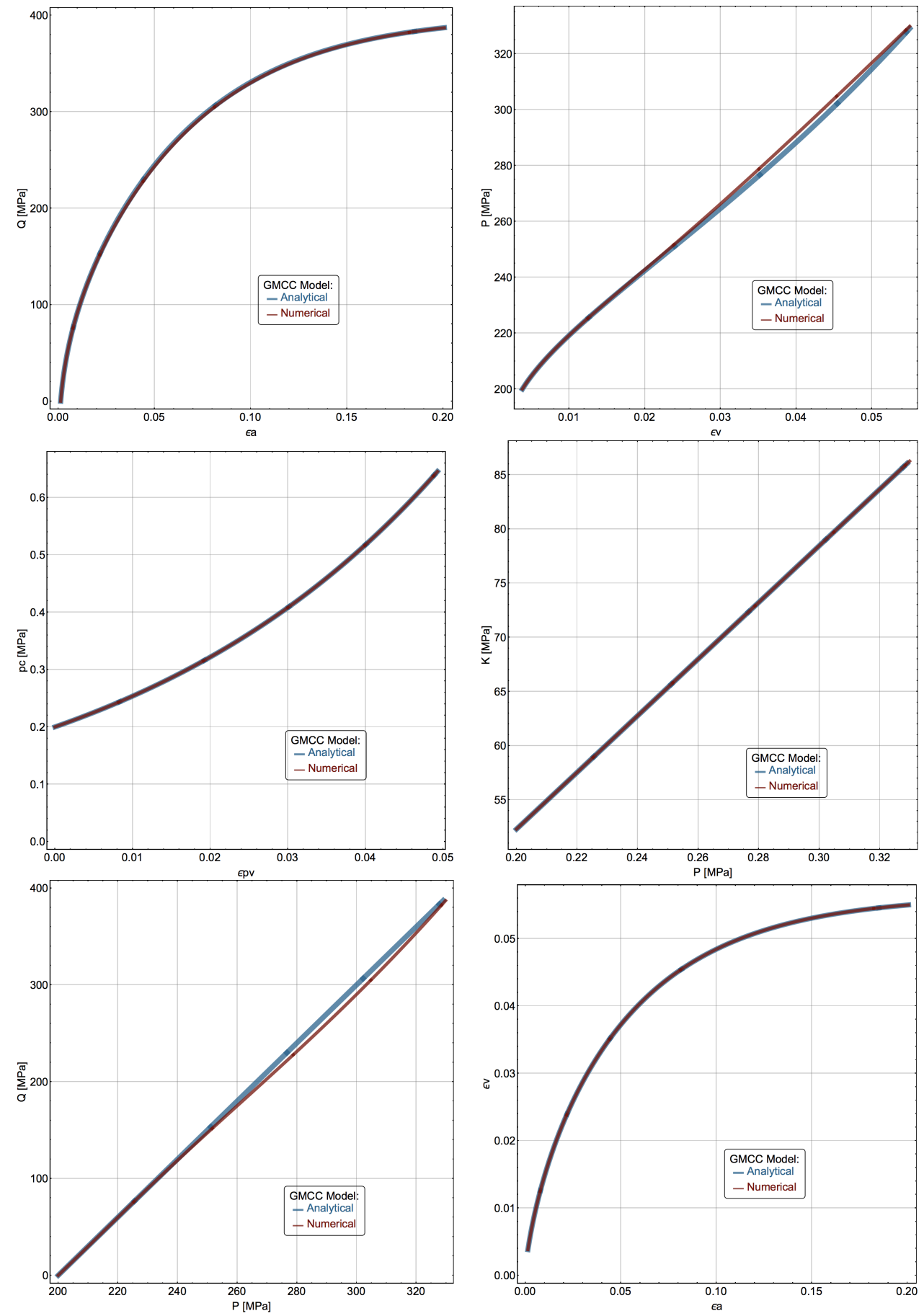

Fig. 5. Comparison between the numerical results of the GMCC model for constant shear modulus with the analytical solution provided by Perić [18. Relation between axial strain and von Mises stress (top-left), relation between volumetric strain and effective mean stress (top-right), relation between volumet 23 plastic strain and preconsolidation pressure (middle-left), relation between effective mean stress and Bulk modulus (middle-right), relation between effective mean stress and von Mises stress (bottom-left), and relation between axial strain and volumetric strain (bottom-right). 
Fig. 6 (left) shows the evolution of the residual normal when solving the nonlinear systems of equations to project $\sigma_{\text {trial }}^{*}$ on $S_{h}^{*}(a)$ for the $G M C C$ model with constant shear modulus, illustrating that the number of iterations required for convergence is less than 5. A typical 3D profile of the $G M C C$ model for constant shear modulus is shown in Fig. 6 (right).

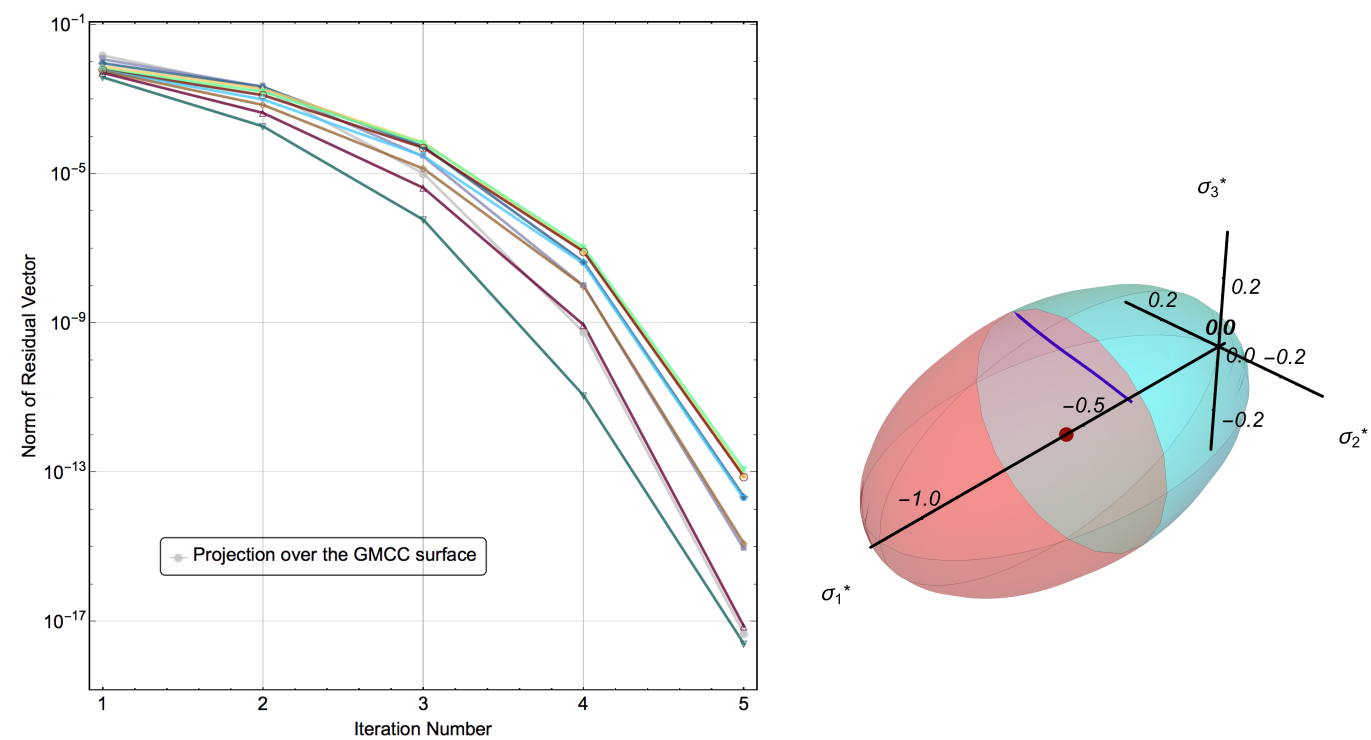

Fig. 6. (Left) Residual norm per iteration for each load step for the GMCC model with constant shear modulus; (Right) The generalized modified Cam-Clay plasticity yield function for constant shear modulus in which the arrow with a blue color shows the evolution of elastoplastic model.

\subsubsection{Verification for constant Poisson's ratio}

All the subfigures in the Fig. 7 illustrates a comparison for the GMCC model implementation with the results calculated from an analytical solution (see in the Appendix D), demonstrating the verification of the implementation for constant Poisson's ratio. 

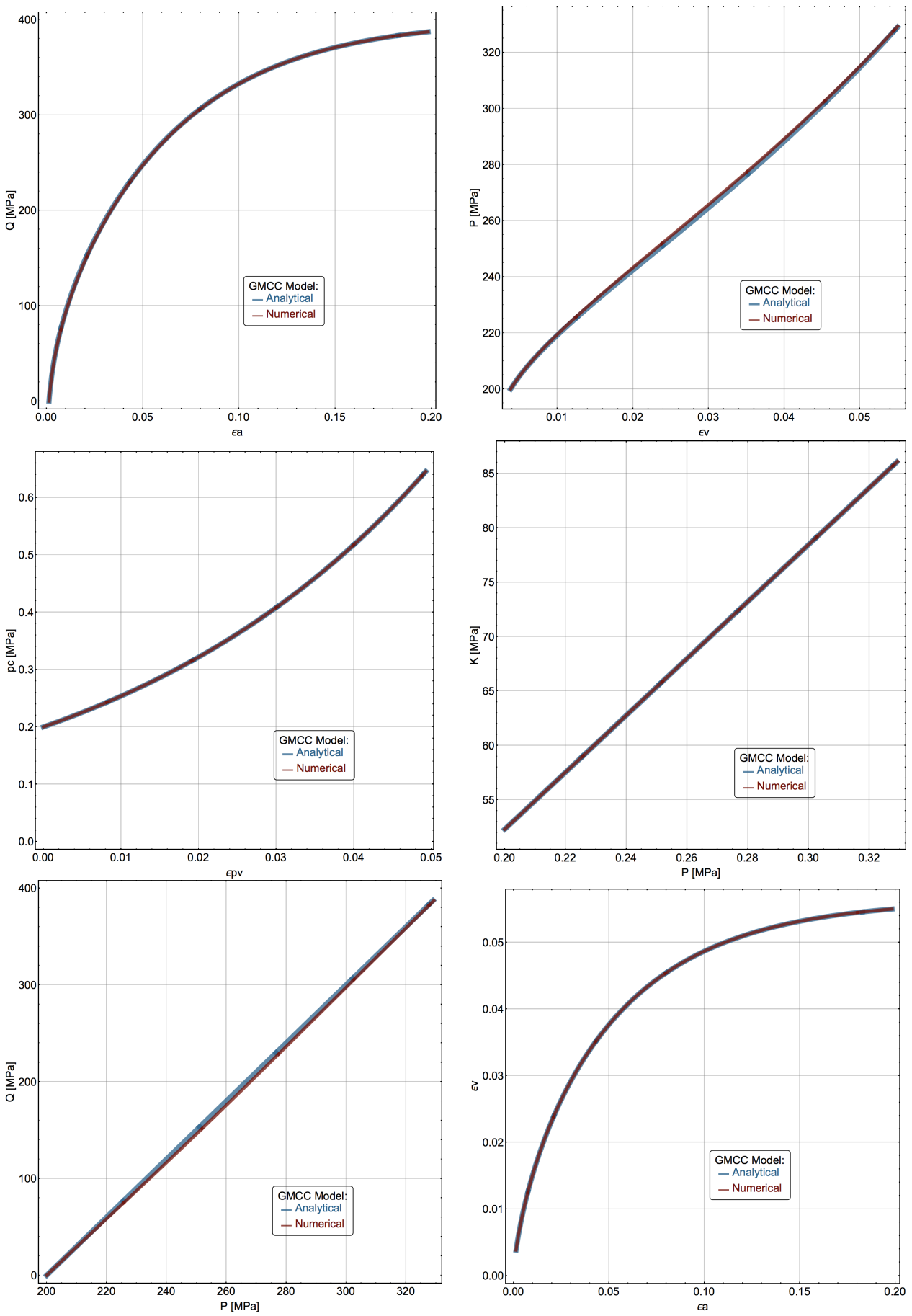

Fig. 7. Comparison between the numerical results of the GMCC model for constant Poisson's ratio with the analytical solution provided by Perić [18. Relation between axial strain and von Mises stress (top-left), relation between volumetric strain and effective mean stress (top-right), relation between volumet 25 plastic strain and preconsolidation pressure (middle-left), relation between effective mean stress and Bulk modulus (middle-right), relation between effective mean stress and von Mises stress (bottom-left), and relation between axial strain and volumetric strain (bottom-right). 
Fig. 8 (left) displays that the evolution of the residual normal when solving the nonlinear systems of equations to project $\sigma_{\text {trial }}^{*}$ on $S_{h}^{*}(a)$ for the $G M C C$ model with constant shear modulus, showing that the number of iterations required for convergence is less than 5 . A typical 3D profile of the $G M C C$ model for constant Poisson's ratio is shown in Fig. 8 (right).

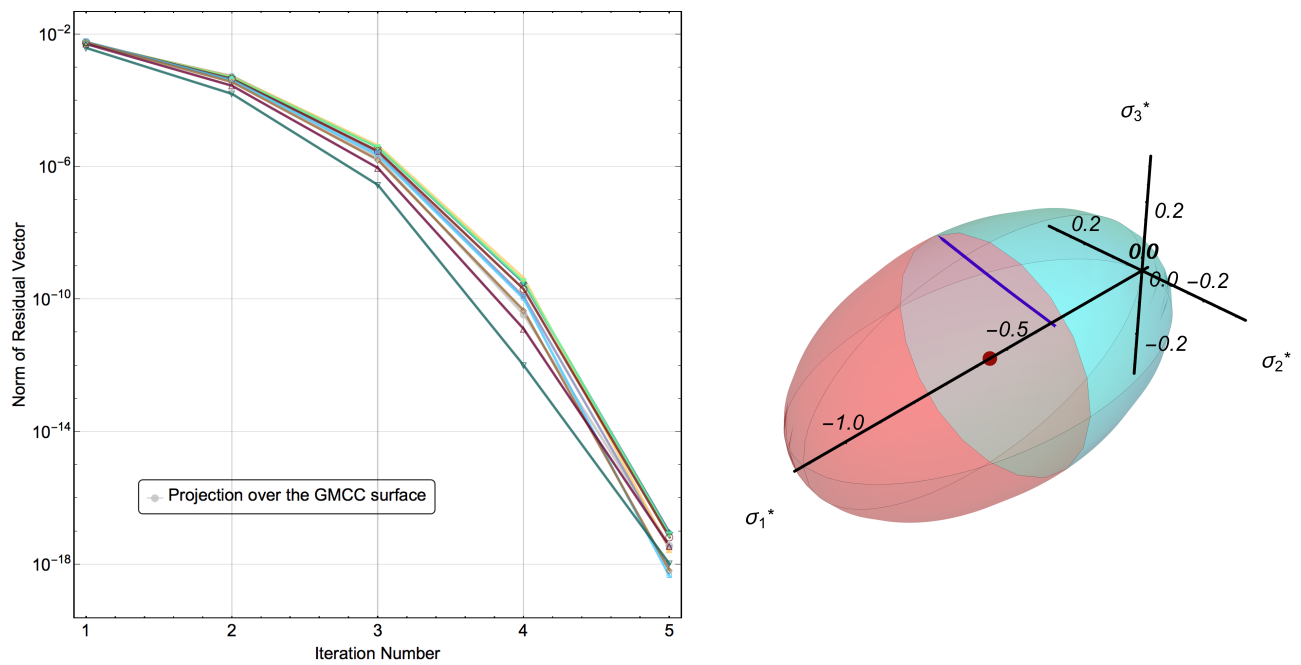

Fig. 8. (Left) Residual norm per iteration for each load step for the GMCC model with constant Poisson's ratio; (Right) The generalized modified Cam-Clay plasticity yield function for constant Poisson's ratio in which the arrow with a blue color shows the evolution of elastoplastic model.

\section{Conclusions}

An innovative description for the modified Cam-Clay plasticity model is developed. The description allows to contemplate the dependence on the Lode's angle. The modified Cam-Clay usually described in the $P-Q$ space is reformulated using the rotated Haigh-Westergaard space. By doing so the plastic return-mapping amounts to closest point projection to the yield surface as a function of a only three independent parameters. The advantage of the new scheme for the modified Cam-Clay is its compactness and numerical efficiency. The Newton method applied to the minimization of a convex function which leads to fast convergence and improved numerical stability. The average number of iterations required for convergence is less than 5 . 


\section{Appendix}

\section{Appendix A: Procedure for solving the elasto plastic return map}

It is done by representing the RHW square distance separately for linear and nonlinear elasticity:

The RHW square distance for nonlinear elasticity is:

$$
\begin{gathered}
\delta_{h}\left(\boldsymbol{\sigma}_{\text {trial }}^{*} ; \theta, \beta, a\right)=\frac{\left(\sigma_{\text {trial }, 1}^{*}-\frac{3(a+a b \cos (\theta))}{\sqrt{3}}\right)^{2}}{3 K_{\text {trial }}}+\frac{\left(\sigma_{\text {trial }, 2}^{*}-\cos (\beta) \frac{\sqrt{2} F(a) \sin (\theta)}{\Gamma(\beta)}\right)^{2}}{2 G_{\text {trial }}}+ \\
\frac{\left(\sigma_{\text {trial }, 3}^{*}-\sin (\beta) \frac{\sqrt{2} F(a) \sin (\theta)}{\Gamma(\beta)}\right)^{2}}{2 G_{\text {trial }}}
\end{gathered}
$$

where the expression for $G_{\text {trial }}$ depends on the flavour of the nonlinear elastic law. And,

$$
\begin{gathered}
\text { res }(\theta, a)=\left[I_{1}\left(\boldsymbol{\sigma}_{\text {trial }}^{*}\right)-I_{1}(\theta, a)\right]-3 K_{\text {trial }}\left[\epsilon_{\text {pv }}\left(a_{\text {trial }}\right)-\epsilon_{p v}(a)\right]=0 \\
\boldsymbol{R}_{h}=\left[\begin{array}{c}
\frac{\partial \delta_{h}\left(\boldsymbol{\sigma}_{\text {trial }}^{*} ; \theta, \beta, a\right)}{\partial \theta} \\
\frac{\partial \delta_{h}\left(\boldsymbol{\sigma}_{\text {trial }}^{*} ; \theta, \beta, a\right)}{\partial \beta} \\
\operatorname{res}(\theta, a)
\end{array}\right]
\end{gathered}
$$

and the corresponding tangent matrix is:

$$
\boldsymbol{J}_{h}=\left[\begin{array}{ccc}
\frac{\partial^{2} \delta_{h}\left(\boldsymbol{\sigma}_{\text {trial }}^{*} ; \theta, \beta, a\right)}{\partial \theta^{2}} & \frac{\partial^{2} \delta_{h}\left(\boldsymbol{\sigma}_{\text {trial }}^{*} ; \theta, \beta, a\right)}{\partial \theta \partial \beta} & \frac{\partial^{2} \delta_{h}\left(\boldsymbol{\sigma}_{\text {trial }}^{*} ; \theta, \beta, a\right)}{\partial \theta \partial a} \\
\frac{\partial^{2} \delta_{h}\left(\boldsymbol{\sigma}_{\text {trial }}^{*} ; \theta, \beta, a\right)}{\partial \beta \partial \theta} & \frac{\partial^{2} \delta_{h}\left(\boldsymbol{\sigma}_{\text {trial }}^{*} ; \theta, \beta, a\right)}{\partial \beta^{2}} & \frac{\partial^{2} \delta_{h}\left(\boldsymbol{\sigma}_{\text {trial }}^{*} ; \theta, \beta, a\right)}{\partial \beta \partial a} \\
\frac{\partial \operatorname{res}(\theta, a)}{\partial \theta} & \frac{\partial \operatorname{res}(\theta, a)}{\partial \beta} & \frac{\partial \operatorname{res}(\theta, a)}{\partial a}
\end{array}\right]
$$

Appendix B: Derivative of project stresses with respect to trial stresses

The chain rule for the hardening surface $S_{h}^{*}$ is:

$$
\frac{\partial \boldsymbol{\sigma}_{\text {proj }}}{\partial \boldsymbol{\sigma}_{\text {trial }}}=\frac{\partial \boldsymbol{\sigma}_{\text {proj }}}{\partial(\theta, \beta, a)} \frac{\partial(\theta, \beta, a)}{\partial \boldsymbol{\sigma}_{\text {trial }}}
$$


By taking the derivative of the RHW square distance as:

$$
\boldsymbol{R}_{h}=0
$$

And applying the Taylor expansion on it, which is:

$$
\frac{\partial \boldsymbol{R}_{h}}{\partial \boldsymbol{\sigma}_{\text {trial }}} \delta \boldsymbol{\sigma}_{\text {trial }}+\frac{\partial \boldsymbol{R}_{h}}{\partial(\theta, \beta, a)} \delta(\theta, \beta, a)+O\left(\boldsymbol{\sigma}_{\text {trial }}, \theta, \beta, a\right)=0
$$

By rearranging it:

$$
\begin{gathered}
\frac{\partial(\theta, \beta, a)}{\partial \boldsymbol{\sigma}_{\text {trial }}}=-\left(\frac{\partial \boldsymbol{R}_{h}}{\partial(\theta, \beta, a)}\right)^{-1} \frac{\partial \boldsymbol{R}_{h}}{\partial \boldsymbol{\sigma}_{\text {trial }}} \\
\frac{\partial(\theta, \beta, a)}{\partial \boldsymbol{\sigma}_{\text {trial }}}=-\left(\boldsymbol{J}_{h}\right)^{-1} \frac{\partial \boldsymbol{R}_{h}}{\partial \boldsymbol{\sigma}_{\text {trial }}} \\
\frac{\partial \boldsymbol{R}_{h}}{\partial \boldsymbol{\sigma}_{\text {trial }}}=\left[\begin{array}{ccc}
\frac{\partial^{2} \delta_{h}}{\partial \theta \partial \sigma_{1}} & \frac{\partial^{2} \delta_{h}}{\partial \theta \partial \sigma_{2}} & \frac{\partial^{2} \delta_{h}}{\partial \theta \partial \sigma_{3}} \\
\frac{\partial^{2} \delta_{h}}{\partial \beta \partial \partial \sigma_{1}} & \frac{\partial^{2} \delta_{h}}{\partial \beta \partial \sigma_{2}} & \frac{\partial^{2} \delta_{h}}{\partial \beta \partial \sigma_{3}} \\
\frac{\partial \Delta a}{\partial \sigma_{1}} & \frac{\partial \Delta a}{\partial \sigma_{2}} & \frac{\partial \Delta a}{\partial \sigma_{3}}
\end{array}\right] \\
\frac{\partial \boldsymbol{\sigma}_{\text {proj }}}{\partial(\theta, \beta, a)}=\left[\begin{array}{ccc}
\frac{\partial \sigma_{1}}{\partial \theta} & \frac{\partial \sigma_{1}}{\partial \beta} & \frac{\partial \sigma_{1}}{\partial a} \\
\frac{\partial \sigma_{2}}{\partial \theta} & \frac{\partial \sigma_{2}}{\partial \beta} & \frac{\partial \sigma_{2}}{\partial a} \\
\frac{\partial \sigma_{3}}{\partial \theta} & \frac{\partial \sigma_{3}}{\partial \beta} & \frac{\partial \sigma_{3}}{\partial a}
\end{array}\right]
\end{gathered}
$$

The variation of the eigenvectors between $\boldsymbol{\sigma}_{\text {proj }}$ and $\boldsymbol{\sigma}_{\text {trial }}$ is represented by a rotation and its inclusion in the tangent matrix can be demonstrated. The detail of the rotation can be found in [16].

\section{Appendix C: Derivation of elastoplastic tangent operator}

The elastoplastic tangent operator is derived from the derivative of the projected stress tensor with respect to the elastic strain tensor and it is defined by the chain rule as follows:

$$
\mathbb{D}^{e p}=\frac{\partial \boldsymbol{\sigma}_{\text {proj }}}{\partial \boldsymbol{\epsilon}_{e}}=\frac{\partial \boldsymbol{\sigma}_{p r o j}}{\partial \boldsymbol{\sigma}_{\text {trial }}} \frac{\partial \boldsymbol{\sigma}_{\text {trial }}}{\partial \boldsymbol{\epsilon}_{e}}
$$

By straightforward derivation, the elastoplastic tangent operator can be written as:

$$
\frac{\partial \boldsymbol{\sigma}_{\text {proj }}}{\partial \boldsymbol{\epsilon}_{e}}=\left(\sum \frac{\partial \boldsymbol{\sigma}_{p r o j, i}}{\partial \boldsymbol{\sigma}_{t r i a l, j}}\left(x_{i} \otimes x_{i}\right) \otimes\left(x_{j} \otimes x_{j}\right)\right) \boldsymbol{C}
$$


The matrix form of elastoplastic tangent operator is:

$$
\frac{\partial \boldsymbol{\sigma}_{p r o j}}{\partial \boldsymbol{\epsilon}_{e}} \delta \boldsymbol{\epsilon}_{e}=\left(\sum \frac{\partial \boldsymbol{\sigma}_{p r o j, i}}{\partial \boldsymbol{\sigma}_{t r i a l, j}}\left(x_{i} \otimes x_{i}\right) \otimes\left(x_{j} \otimes x_{j}\right)\right) \boldsymbol{C} \delta \boldsymbol{\epsilon}_{e}
$$

where $\boldsymbol{C}$ is the elastic tangent operator. To compute elastic tangent operator for constant Poisson's ratio in equation (18), it is required to consider the following remark:

Remark 1: The Bulk modulus in equation (17) is a function of the elastic volumetric deformation. Inspired by the article [6], to prevent a nonconservative model for constant Poisson's ratio in which energy may not be conserved, we propose to maintain the Bulk modulus constant during the time interval $\left[t^{n-1}, t^{n}\right]$ and between timesteps is updated.

\section{Appendix D: Triaxial test on a normally consolidated clay sample}

The triaxial test results are presented for effective mean stress, von Mises stress, elastic volumetric strain, plastic volumetric strain, volumetric strain, generalized shear elastic strain, generalized shear plastic strain, generalized shear strain, preconsolidation pressure, and Bulk modulus, in order to verify the modified Cam-Clay model. The results are expressed separately for case of constant shear modulus and case of constant Poisson's ratio, as follows:

Triaxial test: results for case of constant shear modulus

Table 2. Triaxial results for case of constant shear modulus.

\begin{tabular}{|c|c|c|c|c|c|c|c|c|c|c|c|c|}
\hline No. & $P$ & $Q$ & $\epsilon_{e v}$ & $\epsilon_{p v}$ & $\epsilon_{v}$ & $\epsilon_{e q}$ & $\epsilon_{p q}$ & $\epsilon_{q}$ & $\epsilon_{a}$ & $\epsilon_{r}$ & $p_{c}$ & $K$ \\
\hline Unit & {$[\mathrm{MPa}]$} & [MPa] & & & & & & & & & {$[\mathrm{MPa}]$} & {$[\mathrm{MPa}]$} \\
\hline 1 & 0.2 & 0 . & -0.00382 & 0 . & -0.00382 & 0 . & 0. & 0. & -0.00127 & -0.00127 & 0.2 & 52.3 \\
\hline 2 & 0.207 & 0.0204 & -0.00395 & -0.00169 & -0.00564 & 0.00034 & 0.000123 & 0.000462 & -0.00234 & -0.00165 & 0.208 & 54.08 \\
\hline 3 & 0.214 & 0.0407 & -0.00408 & -0.00381 & -0.00789 & 0.000679 & 0.000563 & 0.00124 & -0.00387 & -0.00201 & 0.219 & 55.85 \\
\hline 4 & 0.22 & 0.0611 & -0.00419 & -0.00627 & -0.0105 & 0.00102 & 0.0014 & 0.00242 & -0.00591 & -0.00228 & 0.232 & 57.63 \\
\hline 5 & 0.227 & 0.0815 & -0.00431 & -0.00896 & -0.0133 & 0.00136 & 0.00268 & 0.00404 & -0.00846 & -0.0024 & 0.247 & 59.41 \\
\hline 6 & 0.234 & 0.102 & -0.00442 & -0.0118 & -0.0162 & 0.0017 & 0.00445 & 0.00615 & -0.0116 & -0.00233 & 0.265 & 61.18 \\
\hline 7 & 0.241 & 0.122 & -0.00453 & -0.0147 & -0.0193 & 0.00204 & 0.00672 & 0.00876 & -0.0152 & -0.00204 & 0.284 & 62.96 \\
\hline 8 & 0.248 & 0.143 & -0.00464 & -0.0177 & -0.0223 & 0.00238 & 0.00953 & 0.0119 & -0.0194 & -0.00149 & 0.305 & 64.73 \\
\hline 9 & 0.254 & 0.163 & -0.00474 & -0.0207 & -0.0254 & 0.00272 & 0.0129 & 0.0156 & -0.0241 & -0.000651 & 0.327 & 66.51 \\
\hline 10 & 0.261 & 0.183 & -0.00484 & -0.0236 & -0.0284 & 0.00306 & 0.0169 & 0.02 & -0.0295 & 0.000506 & 0.351 & 68.29 \\
\hline 11 & 0.268 & 0.204 & -0.00494 & -0.0265 & -0.0314 & 0.0034 & 0.0216 & 0.025 & -0.0355 & 0.00202 & 0.376 & 70.06 \\
\hline 12 & 0.275 & 0.224 & -0.00504 & -0.0293 & -0.0344 & 0.00374 & 0.0271 & 0.0308 & -0.0423 & 0.00395 & 0.402 & 71.84 \\
\hline 13 & 0.281 & 0.244 & -0.00513 & -0.0321 & -0.0372 & 0.00407 & 0.0335 & 0.0375 & -0.0499 & 0.00636 & 0.429 & 73.61 \\
\hline 14 & 0.288 & 0.265 & -0.00522 & -0.0348 & -0.04 & 0.00441 & 0.041 & 0.0454 & -0.0587 & 0.00936 & 0.457 & 75.39 \\
\hline 15 & 0.295 & 0.285 & -0.00531 & -0.0374 & -0.0427 & 0.00475 & 0.0499 & 0.0547 & -0.0689 & 0.0131 & 0.487 & 77.17 \\
\hline 16 & 0.302 & 0.306 & -0.0054 & -0.0399 & -0.0453 & 0.00509 & 0.0609 & 0.0659 & -0.0811 & 0.0179 & 0.517 & 78.94 \\
\hline 17 & 0.309 & 0.326 & -0.00548 & -0.0424 & -0.0479 & 0.00543 & 0.0746 & 0.0801 & -0.096 & 0.0241 & 0.548 & 80.72 \\
\hline 18 & 0.315 & 0.346 & -0.00557 & -0.0448 & -0.0503 & 0.00577 & 0.0931 & 0.0989 & -0.116 & 0.0327 & 0.58 & 82.49 \\
\hline 19 & 0.322 & 0.367 & -0.00565 & -0.047 & -0.0527 & 0.00611 & 0.121 & 0.127 & -0.144 & 0.0458 & 0.612 & 84.27 \\
\hline 20 & 0.329 & 0.387 & -0.00573 & -0.0493 & -0.055 & 0.00645 & 0.176 & 0.182 & -0.201 & 0.0728 & 0.645 & 86.05 \\
\hline
\end{tabular}


Table 3. Triaxial results for case of constant Poisson's ratio.

\begin{tabular}{|c|c|c|c|c|c|c|c|c|c|c|c|c|}
\hline No. & $P$ & $Q$ & $\epsilon_{e v}$ & $\epsilon_{p v}$ & $\epsilon_{v}$ & $\epsilon_{e q}$ & $\epsilon_{p q}$ & $\epsilon_{q}$ & $\epsilon_{a}$ & $\epsilon_{r}$ & $p_{c}$ & $K$ \\
\hline Unit & [MPa] & [MPa] & & & & & & & & & {$[\mathrm{MPa}]$} & [MPa] \\
\hline 1 & 0.2 & 0 . & -0.00382 & 0 . & -0.00382 & 0. & 0. & 0. & -0.00127 & -0.00127 & 0.2 & 52.3 \\
\hline 2 & 0.207 & 0.0204 & -0.00395 & -0.00169 & -0.00564 & 0.000277 & 0.000123 & 0.000399 & -0.00228 & -0.00168 & 0.208 & 54.08 \\
\hline 3 & 0.214 & 0.0407 & -0.00408 & -0.00381 & -0.00789 & 0.000544 & 0.000563 & 0.00111 & -0.00374 & -0.00208 & 0.219 & 55.85 \\
\hline 4 & 0.22 & 0.0611 & -0.00419 & -0.00627 & -0.0105 & 0.000804 & 0.0014 & 0.0022 & -0.00569 & -0.00239 & 0.232 & 57.63 \\
\hline 5 & 0.227 & 0.0815 & -0.00431 & -0.00896 & -0.0133 & 0.00106 & 0.00268 & 0.00374 & -0.00816 & -0.00255 & 0.247 & 59.41 \\
\hline 6 & 0.234 & 0.102 & -0.00442 & -0.0118 & -0.0162 & 0.0013 & 0.00445 & 0.00575 & -0.0112 & -0.00253 & 0.265 & 61.18 \\
\hline 7 & 0.241 & 0.122 & -0.00453 & -0.0147 & -0.0193 & 0.00154 & 0.00672 & 0.00826 & -0.0147 & -0.00229 & 0.284 & 62.96 \\
\hline 8 & 0.248 & 0.143 & -0.00464 & -0.0177 & -0.0223 & 0.00177 & 0.00953 & 0.0113 & -0.0187 & -0.00179 & 0.305 & 64.73 \\
\hline 9 & 0.254 & 0.163 & -0.00474 & -0.0207 & -0.0254 & 0.00199 & 0.0129 & 0.0149 & -0.0234 & -0.00101 & 0.327 & 66.51 \\
\hline 10 & 0.261 & 0.183 & -0.00484 & -0.0236 & -0.0284 & 0.00221 & 0.0169 & 0.0191 & -0.0286 & 0.0000824 & 0.351 & 68.29 \\
\hline 11 & 0.268 & 0.204 & -0.00494 & -0.0265 & -0.0314 & 0.00242 & 0.0216 & 0.024 & -0.0345 & 0.00153 & 0.376 & 70.06 \\
\hline 12 & 0.275 & 0.224 & -0.00504 & -0.0293 & -0.0344 & 0.00263 & 0.0271 & 0.0297 & -0.0412 & 0.0034 & 0.402 & 71.84 \\
\hline 13 & 0.281 & 0.244 & -0.00513 & -0.0321 & -0.0372 & 0.00283 & 0.0335 & 0.0363 & -0.0487 & 0.00574 & 0.429 & 73.61 \\
\hline 14 & 0.288 & 0.265 & -0.00522 & -0.0348 & -0.04 & 0.00303 & 0.041 & 0.044 & -0.0573 & 0.00867 & 0.457 & 75.39 \\
\hline 15 & 0.295 & 0.285 & -0.00531 & -0.0374 & -0.0427 & 0.00322 & 0.0499 & 0.0532 & -0.0674 & 0.0123 & 0.487 & 77.17 \\
\hline 16 & 0.302 & 0.306 & -0.0054 & -0.0399 & -0.0453 & 0.00341 & 0.0609 & 0.0643 & -0.0794 & 0.017 & 0.517 & 78.94 \\
\hline 17 & 0.309 & 0.326 & -0.00548 & -0.0424 & -0.0479 & 0.0036 & 0.0746 & 0.0782 & -0.0942 & 0.0232 & 0.548 & 80.72 \\
\hline 18 & 0.315 & 0.346 & -0.00557 & -0.0448 & -0.0503 & 0.00378 & 0.0931 & 0.0969 & -0.114 & 0.0317 & 0.58 & 82.49 \\
\hline 19 & 0.322 & 0.367 & -0.00565 & -0.047 & -0.0527 & 0.00395 & 0.121 & 0.125 & -0.142 & 0.0448 & 0.612 & 84.27 \\
\hline 20 & 0.329 & 0.387 & -0.00573 & -0.0493 & -0.055 & 0.00412 & 0.176 & 0.18 & -0.198 & 0.0716 & 0.645 & 86.05 \\
\hline
\end{tabular}

\section{Acknowledgements}

The authors M. Sanei, O. Durán, P.R.B. Devloo thankfully acknowledge financial support from ANP-Brazilian National Agency of Petroleum, Natural Gas and Biofuels (ANP-PETROBRAS) (grant 2014/00090-2). P.R.B. Devloo also acknowledges financial support from FAPESP, Brazil - Fundação de Amparo à Pesquisa do Estado de São Paulo, Brazil (grant 2017/157363), and from CNPq - Conselho Nacional de Desenvolvimento Científico e Tecnológico (grant 310369/2006-1).

\section{References}

[1] R. Saian, M. A. Abbas (Eds.), Proceedings of the Second International Conference on the Future of ASEAN (ICoFA) 2017 - Volume 2, Springer Singapore, 2018.

[2] K. Roscoe, A. Schofield, A. Thurairajah, Yielding of clays in states wetter than critical, Géotechnique 13 (1963) 211-240.

[3] A. Schofield, P. Wroth, Critical state soil mechanics, Cambridge University Press (1968). 
[4] K. Roscoe, J. Burland, On the generalized stress-strain behaviour of wet clay, in: J. Heyman, F.A. Leckie (Eds.) (1968) 535-609.

[5] R. Borja, E. Kavazanjian, A constitutive model for the stress-strain-time behaviour of 'wet' clays, Géotechnique 35 (1985) 283298.

[6] R. I. Borja, S. R. Lee, Cam-clay plasticity, part 1: Implicit integration of elasto-plastic constitutive relations, Computer Methods in Applied Mechanics and Engineering 78 (1990) 49-72.

[7] H. Alawaji, K. Runesson, S. Sture, K. Axelsson, Implicit integration in soil plasticity under mixed control for drained and undrained response, International Journal for Numerical and Analytical Methods in Geomechanics 16 (1992) 737-756.

[8] D. Perić, M. A. Ayari, On the analytical solutions for the three-invariant cam clay model, International Journal of Plasticity 18 (2002) 1061-1082.

[9] R. I. Borja, Cam-clay plasticity, part II: Implicit integration of constitutive equation based on a nonlinear elastic stress predictor, Computer Methods in Applied Mechanics and Engineering 88 (1991) 225-240.

[10] R. I. Borja, C. Tamagnini, Cam-clay plasticity part III: Extension of the infinitesimal model to include finite strains, Computer Methods in Applied Mechanics and Engineering 155 (1998) 73-95.

[11] R. I. Borja, C.-H. Lin, F. J. Montáns, Cam-clay plasticity, part IV: Implicit integration of anisotropic bounding surface model with nonlinear hyperelasticity and ellipsoidal loading function, Computer Methods in Applied Mechanics and Engineering 190 (2001) 3293-3323.

[12] R. I. Borja, Cam-clay plasticity. part v: A mathematical framework for three-phase deformation and strain localization analyses of partially saturated porous media, Computer Methods in Applied Mechanics and Engineering 193 (2004) 5301-5338.

[13] K. Krabbenhoft, A. Lyamin, Computational cam clay plasticity using second-order cone programming, Computer Methods in Applied Mechanics and Engineering 209-212 (2012) 239-249. 
[14] M. Rezania, N. Sivasithamparam, M. M. Nezhad, On the stress update algorithm of an advanced critical state elasto-plastic model and the effect of yield function equation, Finite Elements in Analysis and Design 90 (2014) 74-83.

[15] E. de Souza Neto, D. Peri, D. Owen, Computational Methods for Plasticity, John Wiley Sons, Ltd, 2008.

[16] D. L. Cecílio, P. R. Devloo, S. M. Gomes, E. R. dos Santos, N. Shauer, An improved numerical integration algorithm for elastoplastic constitutive equations, Computers and Geotechnics 64 (2015) 1-9.

[17] W. Chen, D. Han, Plasticity for Structural Engineers, Springer-Verlag, 1988.

[18] D. Perić, Analytical solutions for a three-invariant cam clay model subjected to drained loading histories, International Journal for Numerical and Analytical Methods in Geomechanics 30 (2006) 363-387.

[19] P. R. B. Devloo, PZ: An object oriented environment for scientific programming, Computer Methods in Applied Mechanics and Engineering 150 (1997) 133-153.

[20] P. Devloo, Object oriented tools for scientific computing, Engineering with Computers 16 (2000) 63-72.

[21] K. Hayakawa, S. Murakami, Y. Liu, An irreversible thermodynamics theory for elastic-plastic-damage materials, European Journal of Mechanics - A/Solids 17 (1998) 13-32.

[22] J. Lemaitre, J.-L. Chaboche, Mechanics of Solid Materials, Cambridge University Press, 1990.

[23] J. W. Rudnicki, Fluid mass sources and point forces in linear elastic diffusive solids, Mechanics of Materials 5 (1986) 383-393.

[24] A. Kossa, Exact stress integration schemes for elastoplasticity, Ph.D. thesis, Budapest University of Technology and Economics, 2011.

[25] R. Davis, A. Selvadurai, Plasticity and Geomechanics, Cambridge University Press, 2002. 
[26] D. Systémes, Abaqus/Standard Userś Manual, Version 6.12, Simulia, 2012.

[27] D. Ferreira, DIANA Finite Element Analysis, Userś Manual, Version 10.3, Delftechpark 19a, 2628 XJ Delft, The Netherlands, 2019.

[28] C. Foster, R. Regueiro, A. Fossum, R. Borja, Implicit numerical integration of a three-invariant, isotropic/kinematic hardening cap plasticity model for geomaterials, Computer Methods in Applied Mechanics and Engineering 194 (2005) 5109-5138.

[29] E. Oñate, R. Owen (Eds.), Computational Plasticity, Springer Netherlands, 2007.

[30] R. I. Borja, Plasticity, Springer Berlin Heidelberg, 2013.

[31] F. DiMaggio, I. Sandler, Material model for granular soils, Journal of the Engineering Mechanics Division 97 (1971) 935-950.

[32] E. Lainé, C. Vallée, D. Fortuné, Nonlinear isotropic constitutive laws: choice of the three invariants, convex potentials and constitutive inequalities, International Journal of Engineering Science 37 (1999) 1927-1941.

[33] W.-F. Chen, D.-J. Han, Plasticity for Structural Engineers (J Ross Publishing Classics), J. Ross Publishing, 2007.

[34] F. Armero, A. Perez-Foguet, On the formulation of coloset point projection algorithms in elastoplasticity-part i: The variational structure, International Journal for Numerical Methods in Engineering 53 (2001) 297-329.

[35] A. Perez-Foguet, F. Armero, On the formulation of closest-point projection algorithms in elastoplasticity-part ii: Globally convergent schemes, International Journal for Numerical Methods in Engineering 53 (2001) 331-374.

[36] Rocscience, Rocscience users manual, Inc. Toronto, 2015. 